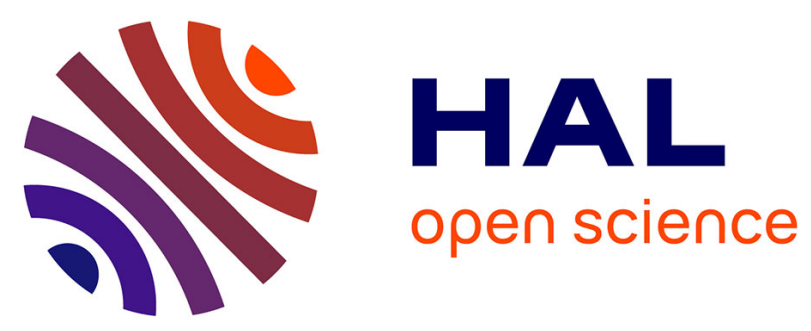

\title{
Eicosapentaenoic acid modulates the synergistic action of CREB1 and ID/E2A family members in the rat pup brain and mouse embryonic stem cells
}

Maurizio Rossi, Martin Spichty, Lucilla Attorri, Chiara Distante, Clara Nervi, Serafina Salvati, Luigi Vitelli

\section{To cite this version:}

Maurizio Rossi, Martin Spichty, Lucilla Attorri, Chiara Distante, Clara Nervi, et al.. Eicosapentaenoic acid modulates the synergistic action of CREB1 and ID/E2A family members in the rat pup brain and mouse embryonic stem cells. Biochimica et Biophysica Acta - Gene Regulatory Mechanisms , 2017, 1860 (8), pp.870-884. 10.1016/j.bbagrm.2017.06.002 . hal-02929345

\section{HAL Id: hal-02929345 \\ https://hal.science/hal-02929345}

Submitted on 17 Nov 2021

HAL is a multi-disciplinary open access archive for the deposit and dissemination of scientific research documents, whether they are published or not. The documents may come from teaching and research institutions in France or abroad, or from public or private research centers.
L'archive ouverte pluridisciplinaire HAL, est destinée au dépôt et à la diffusion de documents scientifiques de niveau recherche, publiés ou non, émanant des établissements d'enseignement et de recherche français ou étrangers, des laboratoires publics ou privés. 
Title. Eicosapentaenoic acid modulates the synergistic action of CREB1 and ID/E2A family members in the rat pup brain and mouse embryonic stem cells Maurizio Rossi ${ }^{\text {a }}$, Martin Spichty ${ }^{b}$, Lucilla Attorri ${ }^{c}$, Chiara Distante ${ }^{a}$, Clara Nervi ${ }^{d}$, Serafina Salvati ${ }^{\mathrm{c}}$, Luigi Vitelli ${ }^{\mathrm{a}, *}$

\section{AFFILIATIONS}

${ }^{a}$ Department of Oncology and Molecular Medicine, Istituto Superiore di Sanità, Viale Regina Elena 299, 00161 Rome, Italy.

${ }^{\mathrm{b}}$ Laboratory of Biology and Modelling of the Cell, Lyon University, ENS Lyon, University Claude Bernard, CNRS UMR 5239, INSERM U1210, 46 allée d'Italie, Site Jacques Monod, F- 69007, Lyon, France.

${ }^{c}$ Department of Public Veterinary Health and Food Safety, Istituto Superiore di Sanità, Viale Regina Elena 299, 00161 Rome, Italy.

${ }^{\mathrm{d}}$ Department of Medical and Surgical Sciences and Biotechnologies, University of Roma "La Sapienza", Corso della Repubblica 79, 04100 Latina, Italy.

* Corresponding author at: Department of Oncology and Molecular Medicine, Istituto Superiore di Sanità, Viale Regina Elena 299, 00161 Rome, Italy.

e-mail: luigi.vitelli@iss.it (L.Vitelli)

Tel. +39-06-49902716

Fax: +39-06-49387087

\section{ABSTRACT}

The aim of this study was to investigate the molecular mechanism by which eicosapentaenoic acid (EPA) may exert neuroprotective effects through an "EPAcyclic AMP response element-binding protein (CREB)" signaling pathway. The current study reveals that EPA modulates the exquisite interplay of interaction of CREB1 with the inhibitor of DNA binding (ID) and E2A family members, thereby 
delivering mechanistic insights into specific neural differentiation program. In this scenario, our work provides evidence for the capability of CREB1 to sequester ID:E2A family members in brain tissues and neural differentiating mouse embryonic stem cells (mESCs) through formation of a [CREB1] 2 :ID2:E47 tetrameric complex. In essence, the molecular function of CREB1 is to dynamically regulate the locationspecific assembly or disassembly of basic-helix-loop-helix (bHLH):HLH protein complexes to mediate the activation of neural/glial target genes. Together, these findings support the one-to-many binding mechanism of CREB1 and indicate that EPA treatment potentiates the integration of CREB dependent signaling with HLH/bHLH transcriptional network, adding specificity to the CREB1-mediated gene regulation during neural/glial differentiation. Our current research on the EPA-CREB axis could reveal new molecular targets for treating neurogenerative disease.

\section{KEYWORDS:}

Lipid signaling; Embryonic stem cell; Eicosapentaenoic acid; Transcription factor CREB; HLH protein; Multiprotein complex

\section{ABBREVIATIONS:}

bHLH, basic-helix-loop-helix; $\mathrm{Ca}^{2+}$, calcium(II) ion; CREB, cyclic AMP response element-binding protein; EPA, eicosapentaenoic acid; GPR40, G-protein coupled receptor 40; ID, inhibitor of DNA binding; LIF, leukemia inhibitory factor; mESC, mouse embryonic stem cells; p-CREB1, CREB1 phosphorylation; PLP, proteolipid protein, PPAR, peroxisome proliferator-activated receptor; PUFAs, polyunsaturated fatty acids; RA, retinoic acid; RXR, retinoid X receptor; t-CREB1, total-CREB1. 


\section{INTRODUCTION}

The benefits of polyunsaturated fatty acids (PUFAs) such as docosahexaenoic acid (22:6n-3) and eicosapentaenoic acid (EPA, 20:5n-3) have been reported in various neurological disorders $[1,2]$. We recently showed that diets enriched with EPA ameliorated the clinical course of experimental autoimmune encephalomyelitis induced in Dark Agouti rats by both triggering myelin repair mechanism and modulating the autoimmune response [2]. In addition, we found that EPA prevents total brain and myelin damage in the cuprizone rat model of multiple sclerosis based on the selective toxicity for oligodendrocytes [3]. However, the underlying mechanisms responsible for the beneficial effects of PUFAs are not well clarified. The neuroprotective effects of EPA and docosahexaenoic acid may result from a variety of overlapping mechanisms including modulation of membrane fluidity and permeability $[4,5]$, alterations in pro- and anti-inflammatory response $[6,7]$, and regulation of transcription factor activities and gene expression [8-10]. Interestingly, non-esterified EPA and docosahexaenoic acid may regulate gene expression via retinoid $X$ receptor $(\mathrm{RXR})$ and peroxisome proliferator-activated receptor (PPAR) [11-13].

In C6 glioma cells the effects of EPA treatment on myelin gene expression appeared more pronounced than those of docosahexaenoic acid and these effects are likely mediated via cyclic AMP-dependent pathway [14]. In vivo studies indicate that early events involving CREB1 phosphorylation (p-CREB1) are coincident with the activation of myelin gene expression in EPA treated rat pup brain [15]. Concordantly, an interesting "PUFA-G-protein coupled receptor 40 (GPR40)- CREB" signaling pathway has been hypothesized for adult primate neurogenesis [16]. Probably distinct signaling pathways control specific temporal phases of p-CREB1 into specific gene expression programs [17]. However, p-CREB1 alone is not sufficient for target gene activation, as shown by genome-wide analysis of CREB target gene activation [18]. Only a minor part of signals that promote p-CREB1 may induce gene activation [19]. A physiologically relevant mechanism could be CREB1 interaction with regulatory 
partners for stable recruitment of the transcriptional apparatus to the promoter of selective CREB1 target genes. The identification of these regulatory partners may allow the determination of the potential contribution of CREB1 interacting factors and their activation in the regulation of stage-specific and signal-dependent developmental responses in immature or mature neural/glial cells from different brain regions.

Recent studies have suggested that PUFA enhances rat neural stem cells by regulating bHLH transcription factors [20]. Several lines of evidence suggest that ID family members, categorized as Class V HLH factors [21], are involved in selfrenewal, proliferation and adhesion to the niche of neural stem cells during development $[22,23]$. Interestingly, a recent work suggests a more sophisticated role of Class I bHLH (E proteins) such as E12 and E47 binding partners of ID proteins in orchestrating neural stem cell differentiation [24]. However the precise mechanism behind $\mathrm{E}$ proteins regulation in differentiating neural stem cells is currently unknown.

The CRE-DNA binding complexes and the protein partners of CREB1 were investigated in the rat pup brain and $\mathrm{mESCs}$ before and after EPA treatment. Our results suggest that EPA stimulates neural/glial differentiation by modulating the levels of HLH/bHLH transcription factors and promoting a direct cross-talk between CREB1 isoforms and HLH/bHLH family members. We propose that a [CREB1 $]_{2}:$ ID2:E47 tetrameric complex plays a key role in an EPA-CREB signaling pathway during neural differentiation. The identification of ID2 and E2A as regulatory partners of CREB1 in brain tissues and retinoic acid (RA) differentiating mESC, reveals new insights to explain how CREB1 can elicit distinct and stagespecific transcriptional programs during neurogenesis and in other differentiation process. 


\section{Materials and Methods}

\subsection{Animal Treatment}

All experiments were performed according to the European Community Council Directive of 24 November 1986 (86/609/ECC) and Italian legislation (DL 116/92) for the treatment and handling of animals. The Italian Ministry of Health approved this study (DM 151/2004-B). Sprague-Dawley rats were obtained from a Charles River colony. Litter size was adjusted to eight pups upon parturition. In our previous investigation [15] we calibrated the dose response effect of EPA in stimulating PLP gene expression. We found that PLP gene expression was more pronounced in rats treated with $10 \mathrm{mM}$ EPA. For this reason, all the experiments were carried out under

\subsection{Cell culture} intracerebroventricularly on postnatal day 2 with $3 \mu 1$ of 10 mM EPA (Sigma, St. Louis, MO) in Dulbecco's modified Eagle's high-glucose medium (DMEM; Invitrogen Life Technologies, Bethesda, MD) as described [15, 25]. The four remaining control pups received $3 \mu \mathrm{l}$ of DMEM. At 1h, $6 \mathrm{~h}$, and $24 \mathrm{~h}$ after EPA injection, pups were sacrificed by decapitation. The brains were carefully removed and dissected into medulla, cerebellum, and cerebral cortex. The isolated regions, pooled for each experiment, were quickly frozen in liquid nitrogen for nuclear protein extraction. At least three different litters were analyzed at specific time points.

The feeder-independent mESC line CGR8 was cultered in Glasgow's Modified Eagle's Medium (GMEM) supplemented with 10\% fetal calf serum, $2 \mathrm{mM} \mathrm{L-}$ glutamine, $1 \%$ nonessential amino acid solution, $1 \mathrm{mM}$ sodium pyruvate, $0.1 \mathrm{mM} 2-$ mercaptoethanol (Sigma), $100 \mathrm{U} / \mathrm{mL}$ penicillin, $100 \mathrm{mg} / \mathrm{mL}$ streptomycin, and 1000 $\mathrm{U} / \mathrm{mL}$ human leukemia inhibitory factor (LIF) as previously described [26]. ESC differentiation in monolayer was induced by withdrawal of LIF for $24 \mathrm{~h}$, followed by treatment with $10^{-6} \mathrm{M}$ RA for $24 \mathrm{hr}$. In some experiments, $10 \mathrm{mM}$ EPA was added to 
the complete ESC medium in the LIF or RA treated ESC culture and the cells were collected at the indicated time points.

\subsection{Immunoblotting}

The nuclear extracts from cortex, cerebellum, and medulla were obtained as previously reported [15]. The different brain regions were homogenized in a buffer containing $10 \mathrm{mM}$ sodium HEPES (pH 7.9), $10 \mathrm{mM} \mathrm{KCl,} 0.5 \mathrm{mM}$ EDTA, $0.1 \mathrm{mM}$ EGTA, 5 mM DTT, 0,5\% Nonidet P-40 (Sigma-Aldrich, Saint Louis, MO) and protease inhibitor cocktail (Sigma-Aldrich).

In addition, the nuclear extract from undifferentiated and RA differentiated ESCs were done as previously described [27]. The protein concentrations of all extracts were determined by the Bradford assay (Bio-Rad Laboratories, Hercules, CA, USA). Proteins (20-25 $\mu$ g per lane) were resolved by SDS-PAGE, and electroblotted onto nitrocellulose membranes for $2 \mathrm{hr}$ at $4^{\circ} \mathrm{C}$. After blocking with 5\% nonfat dry milk in TBST (20 mM Tris- $\mathrm{HCl}, 150 \mathrm{mM} \mathrm{NaCl}, 0.1 \%$ Tween-20) overnight at $4^{\circ} \mathrm{C}$, the blots were incubated with specific primary antibodies at different incubation time from $2 \mathrm{hr}$ to overnight depending on the antibody used. Primary antibodies for immunoblotting were: CREB1 (catalog number 05-767; Upstate), p-CREB $\left(\operatorname{Ser}^{133}\right.$ ) (catalog number 06-519; Upstate) CREB (catalog number 9197; Cell Signaling Technologies), pCREB $\left(\operatorname{Ser}^{133}\right.$ ) (catalog number 9198; Cell Signaling Technologies) and Id1 (C20) (catalog number sc-488), Id2 (C20) (catalog number sc-489), Id3 (C20) (catalog number sc-490), Id4 (L20) (catalog number sc-491), E12 (H208) (catalog number sc762), E47 (N-649) (catalog number sc-763) (all from Santa Cruz Biotecnology).

Secondary anti-mouse or anti-rabbit antibody coupled to horseradish peroxidase were from Bio-Rad Laboratories. Positive signals were revealed by an enhanced chemiluminescence (ECL) detection kit (SuperSignal Substrate, Pierce) according to the manufacturer's instructions. After being stripped, the membranes were reprobed with anti-actin monoclonal antibody (Chemicon, Temecula, CA). Immunoblots were quantified by densitometry using the Bio-Rad Gel Doc 2000. 
For coimmunoprecipitation, primary antibody and normal rabbit serum were coupled to protein A or G Dynabeads (Invitrogen) and then incubated with nuclear extracts prepared from rat pup brain or ESCs. The lysate-beads/antibody conjugate mixtures were incubated overnight at $4{ }^{\circ} \mathrm{C}$ with gentle circular rocking. Immunoprecipitated proteins were eluted from the beads using a magnetic particle concentrator and then subjected to SDS-PAGE and immunoblotting. CREB1 (catalog number 05-767; Upstate), Id2 (catalog number sc-489; Santa Cruz Biotechnology) and E47 (catalog number sc-763; Santa Cruz Biotecnology) antibodies were used for coimmunoprecipitation.

\subsection{EMSA}

Electrophoretic mobility shift assay (EMSA) was performed as previously reported [27]. Each $20 \mu \mathrm{l}$ of reaction mixture contained 10-25 $\mu \mathrm{g}$ of nuclear extract, 10 mM HEPES (pH 7.9), 50 mM KCl, 2 mM MgCl2, 4\% Ficoll, 1 mM EDTA, 1 mM DTT, $0.5 \mu \mathrm{g}$ of poly (dI-dC), and 1-3 pmol of a $\left[\gamma_{-}{ }^{32} \mathrm{P}\right]$ ATP -labeled double-stranded oligonucleotide probe containing the CRE consensus sequence (underlined), 5' GGCTGTAGAATTTGACGTCATCACTCATTACACTGA-3'(top strand). After $15 \mathrm{~min}$ of incubation at room temperature, the assay mixture was loaded onto a $4 \%$ polyacrylamide gel containing $0.25 \mathrm{TBE}$ ( $25 \mathrm{mM}$ Tris-borate, $1 \mathrm{mM}$ EDTA) as the running buffer and electrophoresed at $180 \mathrm{~V}$ for $4-5 \mathrm{hr}$ at $4^{\circ} \mathrm{C}$. Free probe was run off the bottom of gels to facilitate the clear identification of high molecular weight shifted bands.

In some binding reactions, the extracts were preincubated for 10-15 min at room temperature with 1-2 $\mu$ of one of the following reagents: CREB1 (catalog number 05-767; Upstate), p-CREB1 ( $\operatorname{Ser}^{133}$ ) (catalog number 06-519; Upstate) CREB (catalog number 9197; Cell Signaling Technologies), p-CREB $\left(\operatorname{Ser}^{133}\right)$ (catalog number 9198; Cell Signaling Technologies), anti-Cyclin D1 (catalog number 556470, 
BD Biosciences), normal rabbit serum (Bio-Rad Laboratories) and Id1 (C20X) (catalog number sc-488X), Id2 (C20X) (catalog number sc-489X), Id3 (C20X) (catalog number sc-490X), Id4 (L20X) (catalog number sc-491X), E12 (H208X) (catalog number sc-762X), E47 (N-649X) (catalog number sc-763X), ATF3 (C19X) (catalog number sc-188X), ATF4 (also designated CREB-2) (C20X) (catalog number sc-200X), control IgGs (all from Santa Cruz Biotecnology).

From 50 to 300-fold molar excess of CRE or non CRE-containing OCT-1 (top strand 5' - TGTCGAATGCAAATCACTAGAA -3') cold oligonucleotide competitor (Oct1

\subsection{Chromatin immunoprecipitation (ChIP) assays} box is underlined) was included in some binding reaction mixtures.

Undifferentiated and 24h EPA (10 mM) treated ESCs were cultured as described above. ChIP assays were performed as previously described [28] with minor modifications. Briefly, formaldehyde (Fisher Scientific, Pittsburgh, PA) was added to the culture medium to a final concentration of $1 \%$. For each immunoprecipitation, a total of $2 \times 10^{6}$ of ESCs untreated or treated with EPA were cross-linked and sonicated. Sheared chromatin samples were immunoprecipitated overnight at $4{ }^{\circ} \mathrm{C}$ with antibodies specific for anti-CREB1 (3 $\mu \mathrm{g}$; catalog number 05-767, Upstate) or an irrelevant anti-cyclin B1 antibody (3 $\mu \mathrm{g}$; catalog number sc-752, Santa Cruz Biotechnology). The final DNA pellets were resuspended in ultra-pure H2O (Life Technologies) and subjected to PCR. Samples representing $0.02 \%$ of total chromatin (input) were included in the PCR analysis. A genomic region of $364 \mathrm{bp}$ containing a non canonical CRE binding site in the mouse myelin proteolipid protein (PLP) promoter (GenBank: X66421.1) was amplified by PCR using specific primers flanking the non canonical CRE site (forward)(957/977 bp) 5' TTTTGAGCCTGGTCACACACAG3' and reverse (1301/1321 bp) 5'GGATTCAAGAACCCCTCCAT3' and PCR conditions: $94^{\circ} \mathrm{C}$ for $5 \mathrm{~min}$; 40 cycles of $\left(95^{\circ} \mathrm{C}\right.$ for $60 \mathrm{~s} ; 58^{\circ} \mathrm{C}$ for $60 \mathrm{~s} ; 72^{\circ} \mathrm{C}$ for $\left.90 \mathrm{~s}\right)$ and $72^{\circ} \mathrm{C}$ for $5 \mathrm{~min}$. PCR products were analysed by Southern blot analysis and hybridized with an internal ${ }^{32} \mathrm{P}-$ labeled 
oligonucleotide probe containing the putative CRE-like sequence (underlined) in the mouse myelin promoter $(1239 / 1268 \mathrm{bp})$ :

5'CATGACTTTACATGCTCAGACCCAGGTATG3'. The Southern blot analysis and hybridization was performed as previously described [29]. ChIP experiments were repeated three times with similar results.

\subsection{Building of structural model}

We used the CREB1 basic leucine zipper domain (residues 285-339) and the helix-loop-helix domains of ID2 (residues 30-88) and E47 (residues 543-602) to build a model for the interaction of CREB1 and ID2:E47 heterodimer. The basic leucine zipper domain is crucial for CREB1 DNA binding, dimerization and interactions with transcriptional coactivators (e.g. CRTC2) [30]. The HLH domain of both ID2 and E47 is essential for heterodimer recognition [31, 32]. Starting from the crystal structure of the [E47:NeuroD1 $]_{2}$ tetramer (PDB ID: 2QL2) (Fig. 6A), we replaced by overlay one [E47:NeuroD1] unit by a [CREB $]_{2}:$ DNA unit (PDB ID: 1DH3); and substituted NeuroD1 by ID2 (PDB ID: 4AYA) in the other [E47:NeuroD1] unit using the program PyMol (The PyMOLGraphics System 2002, DeLano Scientific, Palo Alto, CA, USA [http://www.pymol.org]).

After visual inspection of this initial model, we noted a small cleft between the CREB1 homodimer and the ID2:E47 unit. We tested therefore if the size of the contact surface (interface) could be increased by a slight relative rotation of the CREB1 homodimer and the ID2:E47 unit around the two axes indicated in Fig. 6A. Using the program CHARMM [33], we performed a 2-dimensional scan that monitored the size of the interface when the two rotational angles are varied from $60^{\circ}$ to $+60^{\circ}$ in steps of $15^{\circ}$. Initial input files for CHARMM were generated with the CHARMM-GUI [34]. The size of the interface between the ID2:E47 unit and the CREB1 homodimer was calculated as follows: 
251 where $A[\mathrm{X}]$ stands for the surface area of complex $\mathrm{X}$. The surface areas were calculated with the program CHARMM (command "COOR SURF") using the atomic radii from all-atom force field c36 and a solvent probe radius of $1.6 \AA$. Supplementary Table 1 lists the size of the interface as a function of the two scanned angles. The function is rather peaked with a clear maximum for $\left(-30^{\circ},-15^{\circ}\right)$. The negative sign indicates a rotation in left-handed direction. Through these relative rotations the size of the interface could be increased by $10 \%$ from 922 to $1012 \AA^{2}$. To judge if a given relative rotation would lead to steric clashes, we also determined the minimal distance between the backbone Calpha atoms of the CREB1 homodimer and the ID2:E47 unit for each combination of rotational angles (see last column in Supplementary Table 1). We consider a minimal distance of less than $3.5 \AA$ as steric clash $[35,36]$. The rotation $\left(-30^{\circ},-15^{\circ}\right)$ slightly increases the minimal distance from 4.2 to $4.9 \AA$. Thus no steric clash is observed for the rotation with maximal interface size. program CHARMM, we first added hydrogen atoms to a given predicted structure with the command HBUILD and then calculated the difference in surface area between the dimer ID2:E47 and the multimer [CREB1] $]_{2}:$ ID2:E47. The calculation of 
the surface area is detailed above. The command "SCALAR WMAIN STAT"

278 permits the extraction of the contribution of two subunits ID2 and E47 to the buried surface area. Note that the sum of these two contributions is slightly different from the value of the interface area because the former (Eq. 2) considers only contributions from ID2 and E47 whereas the latter (Eq. 1) is an average over the contributions of ID2, E47 and the CREB1 homodimer.

\subsection{Molecular dynamics simulations}

The structure of the tetramer [CREB1] 2 :ID2:E47 bound to DNA was simulated with the CHARMM all-atom force field c36 [38] using the program ACEMD [39] with GPU-acceleration (Nvidia GTX). Standard parameters were used if not specified. The liquid phase was mimicked with 46788 explicit TIP3P water molecules and (charge-neutralizing) counter-ions $(\mathrm{CaCl}, 0.15 \mathrm{M})$ in a cubic box with an initial side length of $112 \AA$. Periodic boundary conditions were applied. The cutoff for short-range nonbonded interactions was $9 \AA$; long-range electrostatic interactions were treated with Particle-Mesh Ewald summation with a grid spacing of $0.875 \AA$. The equation of motion was integrated with a time step of $4 \mathrm{fs}$ with constrained hydrogen bonds and a scaling factor of 4 for ACEMD's hydrogen masses redistribution. The temperature of the system was set to $310 \mathrm{~K}$ (Langevin thermostat). During the first $25 \mathrm{~ns}$, the molecular dynamics was carried out at constant temperature and pressure (Berendsen barostat) to equilibrate the volume. From the last $5 \mathrm{~ns}$ of this equilibration phase we determined an average side-length of $113.8 \AA$ for the cubic simulation box. The remaining $500 \mathrm{~ns}$ of molecular dynamics were carried out at constant volume and temperature. Snapshots were saved every 1 ns.

\subsection{Statistical Analysis}

Statistical analysis for biochemical studies was carried out using the Student's ttest, with $\mathrm{P}<0.05$ defined as statistically significant. 
304 To quantify the amount of variation in the contribution of subunits ID2 and E47 to 305 the buried surface area, we calculated the uncorrected sample standard deviation of 306 the set of docking predictions.

\section{RESULTS}

3.1. p-CREB1 levels and CREB1 occupancy on the PLP promoter in ESCs after EPA treatment

312 Our previous results in the rat pup brain indicate that EPA treatment induces a 313 significant decrease in p-CREB1 both in the cortex and in the cerebellum $1 \mathrm{~h}$ post314 injection, whereas no significant difference was found at $16 \mathrm{~h}$ post-injection [15]. We 315 extended this analysis to mESCs, a well-established in vitro model of early 316 development for the identification of EPA-derived signaling molecules with 317 therapeutic potentials [40-42]. Initially, we measured the levels of CREB1 protein at 318 different time points of EPA treatment. Our results show that p-CREB1 significantly 319 increases $3 \mathrm{~h}$ after EPA treatment compared to LIF control sample (Fig. 1A, B). 320 Afterwards, p-CREB1 decreased from $6 \mathrm{~h}$ until $24 \mathrm{~h}$, whereas the levels of total321 CREB1 (t-CREB1) protein were relatively constant from $1 \mathrm{~h}$ to $24 \mathrm{~h}$ after EPA 322 treatment (Fig. $1 \mathrm{~A}, \mathrm{C}$ ). In conclusion EPA up-modulates p-CREB1 at early times of 323 ESCs treatment with a kinetic that differs from that reported in rat pup brains [15]. 

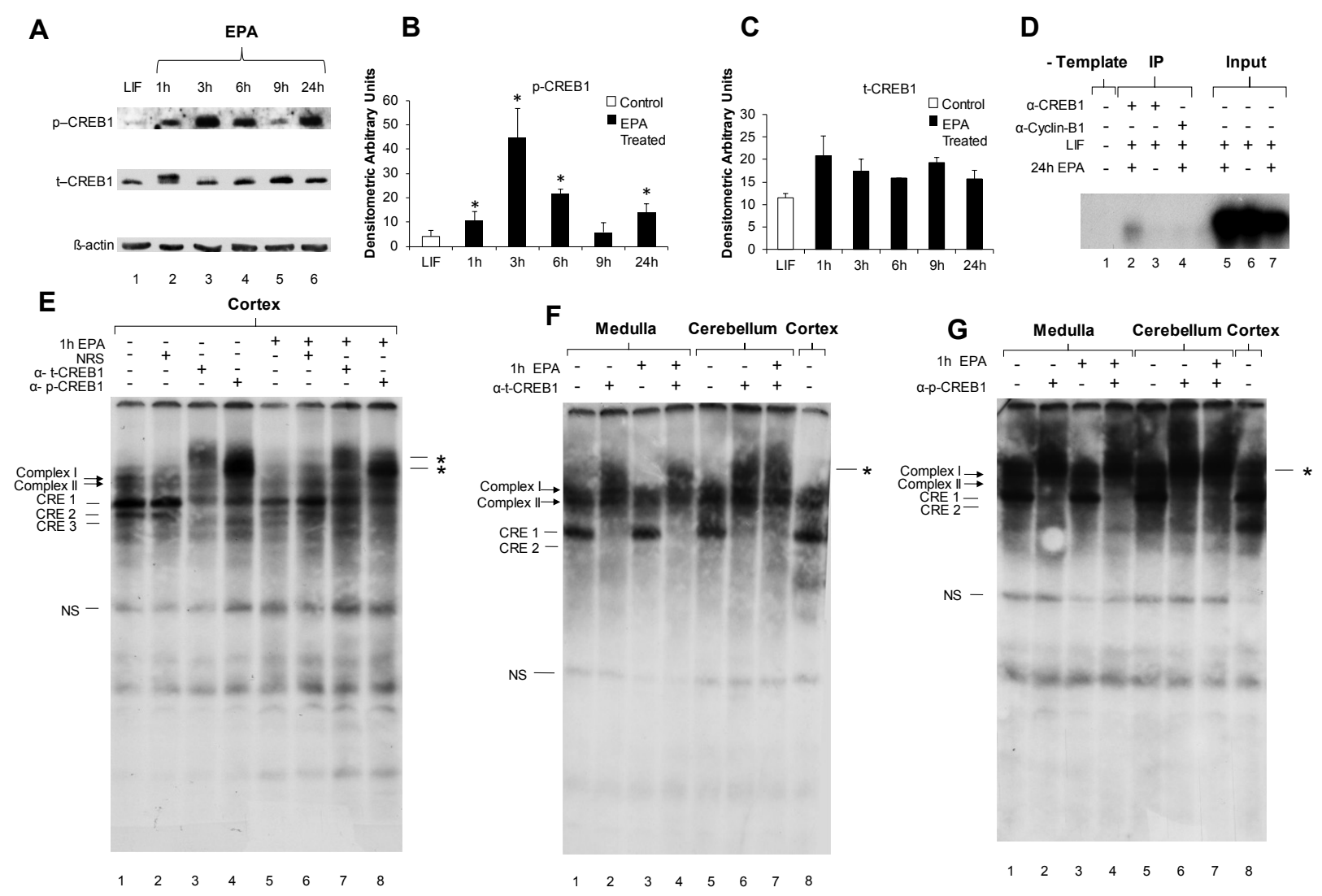

Fig. 1. p-CREB1 levels and CRE-DNA binding activity in mESCs and rat pup brains after EPA treatment. (A) Western blot analysis of t-CREB1 and p-CREB1 in EPA treated ESCs at different time points. (B, C) The levels of p-CREB (B) and t-CREB (C) were analysed by densitometric scanning and reported in the histogram after normalization with $\beta$-actin. Results are mean $\pm \mathrm{SEM}$ of four independent experiments. $* \mathrm{P}<$ 0.05 vs. control. (D) ChIP analysis of CREB1 occupancy on the PLP proximal promoter in EPA treated ESCs. Cross-linked chromatin fragments from LIF-treated (lanes 3,6) or EPA+LIF treated (lanes 2,4,5,7) ESCs were incubated with anti-CREB1 antibodies (lanes 2, 3) or irrelevant anti-cyclin B1 antibodies (lane 4). Immunoprecipitated DNA was analyzed by PCR using primers specific for the mouse myelin PLP promoter. Samples representing $0,02 \%$ of total chromatin were included in the PCR analysis (lanes 5-7). (E to F) Supershift analysis with anti-t-CREB1 and anti-p-CREB1 antibodies in untreated or EPA treated nuclear extracts from the cortex $(\mathrm{E})$, medulla and cerebellum $(F, G)$. Nuclear extracts from the cortex were used as positive control (F, G, lane 8). Asterisks indicate the supershifted complexes. NS, are nonspecific bands; NRS, normal rabbit serum.

CREB1 is an important mediator regulating the expression of myelin-specific genes. Interestingly, a CRE binding site is present in the promoter region of the genes encoding for both myelin PLP and myelin oligodendrocyte glycoprotein [43-45]. In addition, the level of PLP mRNAs increased in nearly all brain regions of EPA 
treated pups [15]. A CRE-like binding sequence 5'-TGACTTCA-3' (instead of the consensus CRE binding site 5'-TGACGTCA-3') is located within the distal footprint FP330 of the rat PLP promoter [43]. In mice, a non-canonical CRE-binding sequence 5'-TGACTTTA-3'(6/8 identical to the canonical CRE) is present at the orthologous CRE site of the rat PLP promoter (see Materials and Methods for details).

To determine whether CREB1 binds to the non-canonical CRE-binding sequence in the PLP promoter, we initially performed ChIP assays in cross-linked chromatin from ESCs untreated or treated with EPA for $24 \mathrm{~h}$ using anti-CREB1antibodies or the unrelated anti-cyclin B1 antibodies used as negative control (Fig.1D). The immunoprecipitates were amplified by PCR using primers flanking the non-canonical CRE-binding sequence in the PLP promoter region and hybridized to the internal 32P-labeled oligonucleotide PLP probe containing the non-canonical CRE site as described in Materials and Methods. Our results showed that CREB1 is recruited on PLP target gene after 24h EPA treatment in ESCs but not in the control sample (Fig. 1D, lanes 2 and 3 respectively). This result confirms that EPA treatment induces the CREB1 occupancy at a non-canonical CRE site in the PLP promoter.

\subsection{The CRE-DNA binding activity in different brain regions of pups after EPA} treatment

To characterize the molecular mechanism of CREB1 transcription function in vivo, we analyzed the CRE-DNA binding activity in different brain regions of pups intracerebroventricularly treated or untreated with EPA. We performed EMSA analysis using a ${ }^{32} \mathrm{P}$-labeled double-stranded oligonucleotide probe containing a canonical CRE consensus sequence. According to our EMSA analysis of nuclear protein extracts from the cortex, the CRE DNA-binding activity is composed of several protein complexes including two high molecular weight complexes (complex I and II), three intermediate molecular weight complexes (CRE1, CRE2 and CRE3), and low molecular weight nonspecific bands (Fig. 1E). One of the most distinct 
nonspecific bands is indicated by NS (Fig. 1E). Sequence specificity of binding was verified by the addition of 50 -fold and 300 -fold molar excess of cold competitor containing the canonical CRE box element (Supplementary Fig. S1, lanes 2 and 3) or with a non-CRE oligonucleotide containing OCT-1 sequence (Supplementary Fig. S1, lane 4). Similar shifts were also observed using medulla and cerebellum nuclear extracts (Fig.1 F and G) indicating the critical role of each one of the CRE-specific DNA-binding complexes in the rat pup brain. Interestingly, the CRE1 is the most abundant intermediate molecular weight complex in all brain regions (Fig.1E, F and G). On the other hand, the CRE3 complex is mainly present in the cortex (Fig. 1 E). Furthermore, the CRE1 and CRE2 complexes are sharply supershifted in the untreated or EPA treated cortex (Fig. 1E, lanes 3 and 7), medulla (Fig. 1F, lanes 2 and 4), and cerebellum (Fig. 1G, lanes 6 and 7) by incubation with an anti t-CREB1 antibody, but not with a normal rabbit serum (Fig. 1E, lanes 2 and 6). Similar results of supershift assays were obtained with an anti-p-CREB1 antibody in the untreated or EPA treated cortex (Fig. 1E, lanes 4 and 8), medulla (Fig. 1F, lanes 2 and 4), and cerebellum (Fig. 1G, lanes 6 and 7) to show that the CRE1 and CRE2 complexes contain an active p-CREB1 protein. Unfortunately, the presence of p-CREB1 was not assessable in the complex I due to an intense supershift band of the anti-p-CREB1 that colocalized within complex I binding region on the autoradiogram masking this complex. Therefore, in different brain regions of pups, at least CRE1 and CRE2 complexes contain canonical p-CREB1 (Ser133) proteins. Probably, the CRE1, CRE2 and CRE3 complexes are generated by dimerization of different splice variants of CREB1 gene as the slower migrating CREB1- $\alpha$ isoform and the faster migrating CREB1 $\Delta$ isoform [46] or by ATF1/CREB1 heterodimerization [47]. However, CREB1 interactions with other as yet unidentified novel partners cannot be excluded. Intriguingly, we found similar shift and supershifts of CRE-DNA binding complexes in untreated or 1h EPA-treated samples of nuclear extracts from different rat pup brain regions (Fig.1 E, F, G). 
3.3. The effect of EPA on the CRE-DNA binding activity in mESCs after LIF withdrawal and RA-induced differentiation

With the aim to better characterize changes in the CRE DNA-binding complex during multi-protein complex formation in early development, we extended our investigation to EPA treated $\mathrm{mESC}$ s that provide a suitable in vitro model for neuralglial differentiation [48]. As shown in Fig. 2A, incubation of a ${ }^{32} \mathrm{P}$-labeled doublestranded oligonucleotide probe containing a CRE-box consensus sequence with LIFtreated mESC extracts generated specific protein-DNA complexes. In particular, complex I and CRE1, CRE2 and CRE3 complexes, were specifically competed by the addition of 50-fold, 150-fold and 300-fold molar excess of cold competitor oligonucleotide containing the canonical CRE sequence (Fig. 2A, lanes 2-5). The higher and lower-mobility complexes were unspecific bands (Fig. 2A, the NS1 and the NS2 band, respectively). In the LIF-treated ESC extracts (Fig. 2A, lane 2), the complex II present in the rat pup brain was not detected and the CRE-1 complex was less abundant compared to the CRE2 and CRE3 complexes. Interestingly, the CRE2 and CRE3 complexes were present in larger quantity in the LIF-treated ESCs when compared to the cortex of rat pup brain (Fig. 2A, lanes 1 and 2). Moreover, the CRE1 complex was more abundant in the cortex than in the undifferentiated ESCs (Fig. 2A, lanes 1 and 2). The complex I, CRE1 and CRE2 complexes were supershifted by incubation with anti-p-CREB1 antibody (Fig. 2B, lane 4) while they were not affected by normal rabbit serum (Fig. 2B, lane 2). In addition, the complex1, CRE1, CRE2 and CRE3 complexes were sharply decreased by incubation with anti-tCREB1 (Fig. 2B, lane 3). As a general comment to our EMSA data, we believe that the disappearance or decrease of a shifted band without causing a supershift can support the identification of the protein of interest when used with the proper controls (e.g. normal rabbit serum). Therefore, our results strongly suggest that p-CREB1 and apparently t-CREB1 are present in the complex I as well as the CRE1, CRE2 and CRE3 complexes in LIF-treated ESCs. 

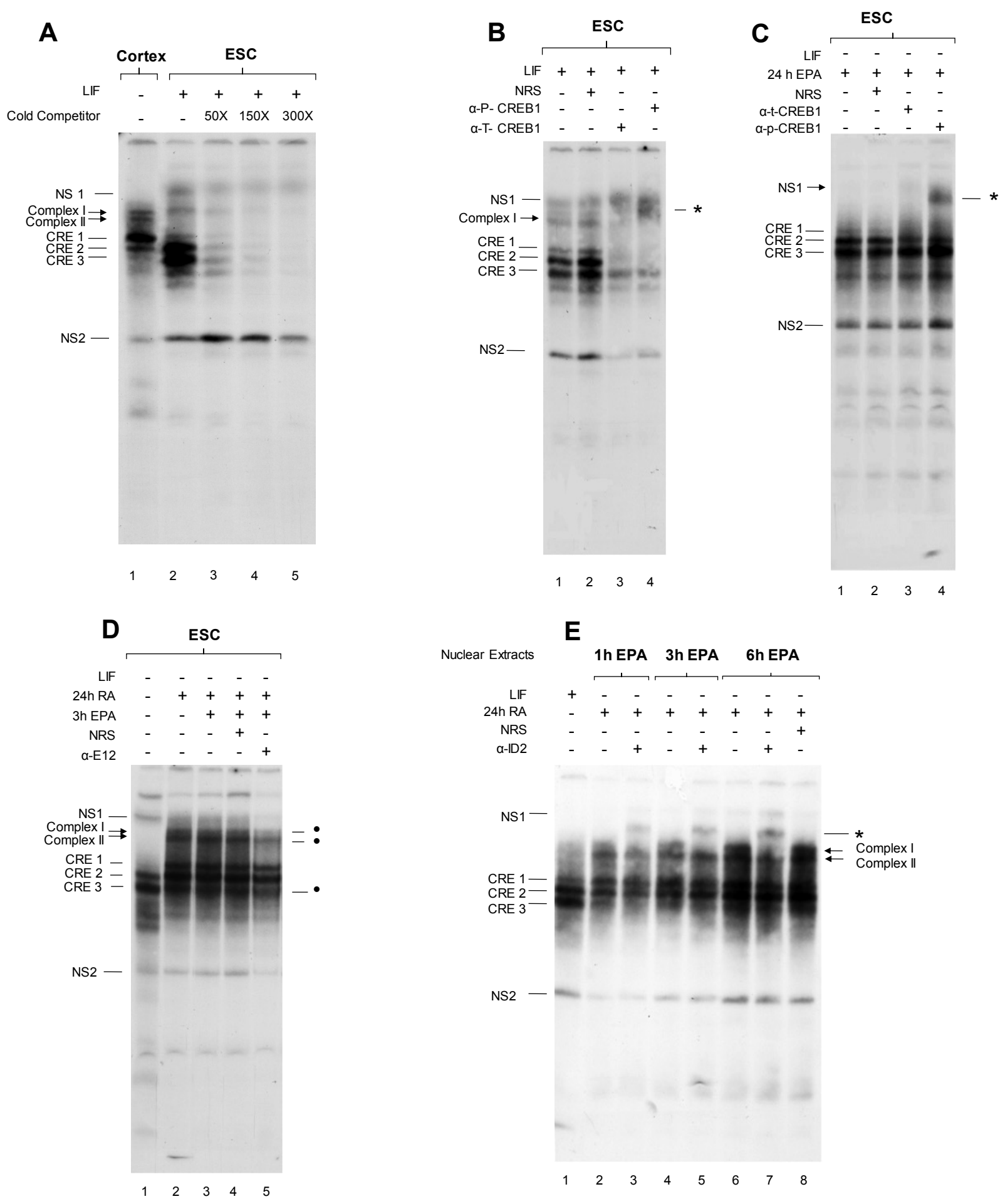

430 Fig. 2. The effect of EPA on the CRE-DNA binding activity in ESCs after LIF withdrawal and RA-induced 431 differentiation. (A) Competition EMSA with 50 to 300-fold molar excesses of cold CRE wild-type 432 oligonucleotides in LIF-treated ESCs. (B, C) Supershift analysis with anti-t-CREB1 and anti-p-CREB1 433 antibodies in LIF-treated ESCs (B) or EPA treated ESCs after LIF withdrawal (C). (D, E) Changes in the 434 profile of CRE DNA-binding activity in ESCs after LIF withdrawal and RA-induced differentiation. EMSA 435 analysis was performed with anti-E12 antibodies (D) and anti-ID2 antibodies (E) in the corresponding EPA plus RA treated samples. Asterisks indicate the supershifted complexes (B, C, E). Dots denote distinct bands 
We next investigated the CRE-DNA binding activity in ESCs treated with EPA for $24 \mathrm{~h}$ after LIF withdrawal. Intringuingly, the high molecular weight complex I and the CRE1 band present in the LIF-treated ESCs, were barely detected in extracts from ESCs treated with EPA for 24h after LIF withdrawal (Fig. 2C, lane 1). On the other hand, the CRE2 and CRE3 complexes were both present in the EPA treated ESCs after LIF withdrawal (Fig. 2C, lane 1). Furthermore, nuclear extracts from EPA treated ESCs were incubated with anti-t-CREB1 and anti-p-CREB1 antibody (Fig. 2C, lanes 3 and 4). The anti-p-CREB1 antibody generates a sharp supershift band of the CRE1 and CRE2 complex (see the dashed line with asterisk in Fig. 2C, lane 4). In addition, the CRE1 and CRE2 complexes were decreased by incubation with anti-tCREB1 antibodies (Fig. 2C, lanes 3 and 4), while they were not affected by normal rabbit serum (Fig. 2C, lane 2). The downmodulation of complex I and CRE1 complex after LIF withdrawal suggests a cross-talk between the LIF/STAT3 and the EPAPPAR/RXR-CREB signaling pathway occurring during ESC self-renewal.

Remarkably, RA-induced differentiation of mESCs has been previously used as a cellular model for studying neural commitment and specification [48-50]. Taking this into account, we investigated the CRE DNA-binding complexes induced by RA in early differentiating mESCs. Outstandingly, the CRE DNA-binding complexes were rapidly changed by RA treatment of mESCs showing a pattern of shift bands very similar to the observed shifted bands in pup brain tissues (Fig. 2, compare lane 1 in panel A with lane 2 in panel D). More precisely, in the mESCs induced to differentiate by RA treatment for $24 \mathrm{~h}$, the complex I and II were successfully reformed and the CRE-1 band was up-modulated when compared to the pattern of CRE-DNA binding complexes observed in the mESCs after LIF withdrawal (Fig. 2C, lanes 1 and 2). In the EPA treated mESCs after $24 \mathrm{~h}$ of RA-induced differentiation, 
complex I and complex II were mainly up-modulated according to the time of EPA treatment (1h, $3 \mathrm{~h}$ and $6 \mathrm{~h}$ ), reaching the highest intensity at $6 \mathrm{~h}$ after treatment (Fig. 2E, lanes 2, 4 and 6).

\subsection{Changes in the level of ID proteins in the rat pup brain and mESCs after EPA} treatment

To determine whether EPA affects ID2 transcription factor activity, the level of ID2 protein was investigated in untreated and EPA treated rat pup brains at $1 \mathrm{~h}, 6 \mathrm{~h}$ and $24 \mathrm{~h}$. We found that at $1 \mathrm{~h}$, the ID2 protein is highly expressed in untreated nuclear extracts from the cortex, less abundant in the cerebellum, and far less expressed in the medulla (Fig 3A). Interestingly, western blot analysis (Fig. 3A) of pup brain nuclear extracts prepared after 1h EPA treatment showed an increase of ID2 protein expression in the cortex, cerebellum and medulla. More precisely, the ID2 protein increased (32\%) in the medulla and in the cerebellum (18\%) as well as in the cortex (18\%) at 1h after EPA treatment (Fig. 3A). Modulation of ID2 levels may represents an early event caused by EPA treatment in the rat pup brain. On the other hand, the ID2 protein decreased in the medulla by $28 \%$ and in the cortex by $25 \%$ after a $6 \mathrm{~h}$ EPA treatment (Fig. 3B). Moreover, we observed a more significant decrease of ID2 in the medulla (49\%) and in the cortex (24\%) after a $24 \mathrm{~h}$ EPA treatment (Fig. 3C). In the cerebellum, no significant differences were found in the ID2 level after $6 \mathrm{~h}$ and $24 \mathrm{~h}$ EPA treatment compared to the control samples (Fig. 3B and C). The early ID2 activation in the pup brain regions was coincident with the decrease of the p-CREB1 activity in the cortex and cerebellum after $1 \mathrm{hr}$ of EPA treatment [15]. 
A

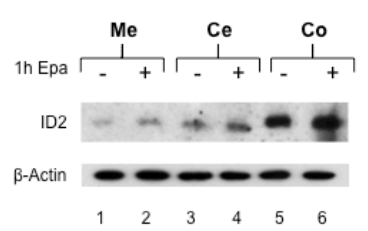

B

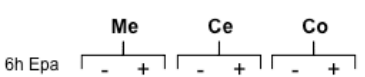

102

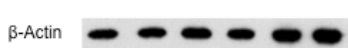

$\begin{array}{llllll}1 & 2 & 3 & 4 & 5 & 6\end{array}$

C

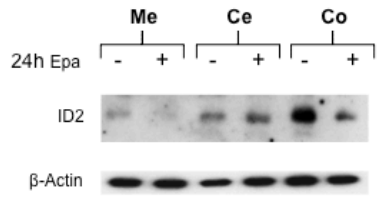

$\begin{array}{llllll}1 & 2 & 3 & 4 & 5 & 6\end{array}$

D

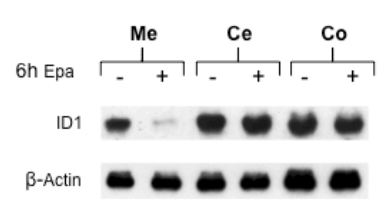

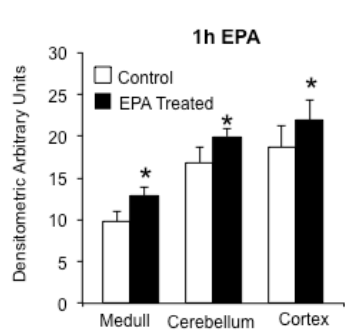
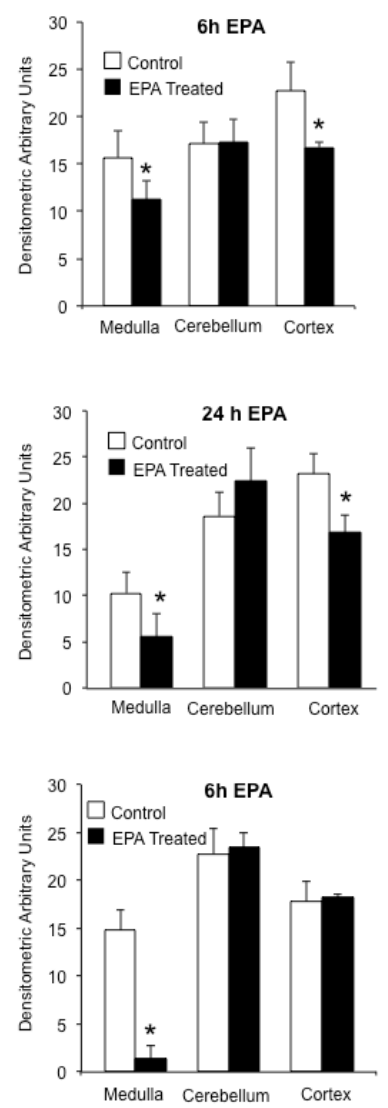

E
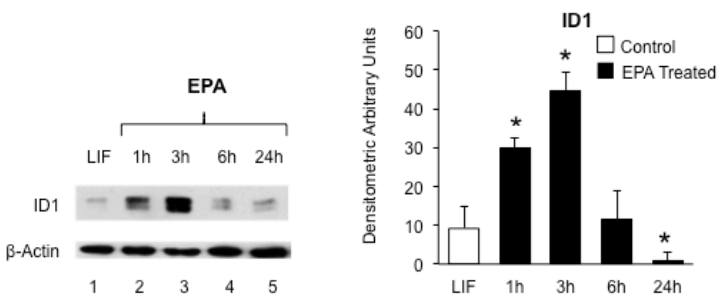

$\mathbf{F}$
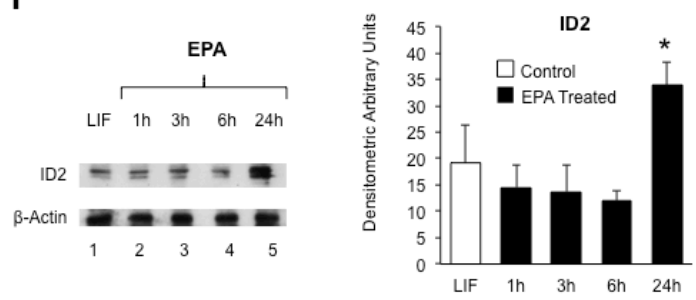

G
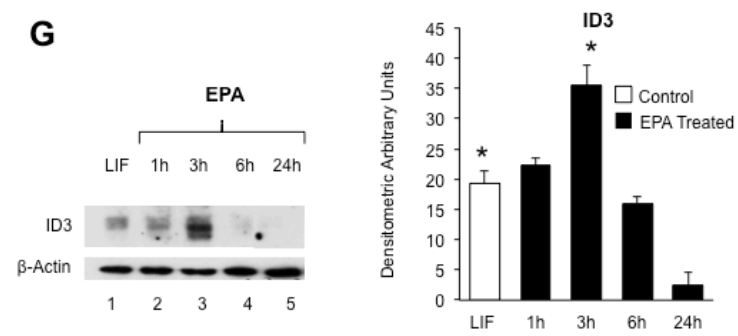

H
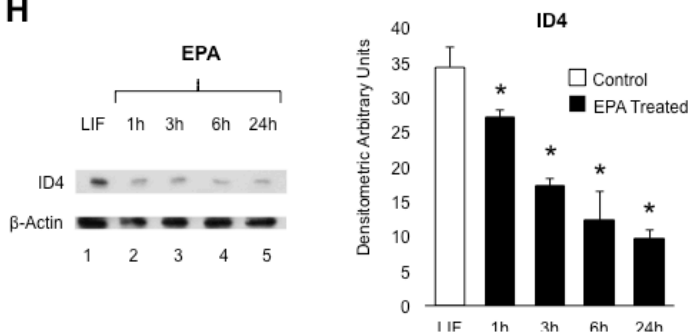

Fig. 3. Changes in the level of ID proteins in the rat pup brain and mESCs after EPA treatment. (A, B, C)

Western blot analysis of ID2 protein in the medulla (lanes 1,2), cerebellum (lanes 3,4) and cortex (lanes 5,6), untreated or treated with EPA after 1h (A), 6h (B) and 24h (C). (D) Western blot analysis of ID1 protein in rat pup brains untreated or treated with EPA after 6h. (E to H) Western blot analysis of ID1 (E), ID2 (F), ID3 (G) and ID4 (H) proteins in mESCs after EPA treatments at different time points. The blots were subsequently re-probed with anti- $\beta$-actin antibodies to monitor equal loading of the samples. ID1, ID2, ID3 and ID4 levels were analyzed in the rat pup brain and mESCs by densitometric scanning and reported in the histogram after normalization to the $\beta$-actin. Values are expressed as mean \pm SEM of three independent experiments. ${ }^{*} \mathrm{P}<0.005$ vs. control. 
to ID2 modulation after EPA treatment, was a very sharp decrease (92\%) of ID1 in the medulla after 6h EPA treatment (Fig. 3D). However, no significant difference of ID1 levels was detected in the cerebellum and in the cortex after 6h EPA treatment (Fig. 3D). The ID1 downregulation by EPA is an interesting finding, which should form the basis for future studies. During brain development, EPA might accelerates the myelinogenesis process in the medulla possibly according to a temporal-spatial pattern of myelination, an hypothesis supported also by findings reported in [15].

Importantly, it has been well established that ID proteins are involved in the $\mathrm{BMP} / \mathrm{SMAD}$ pathway that plays a key role in ESC self-renewal [51]. To better evaluate the EPA effect on ID proteins activity, the levels of ID1, ID2, ID3 and ID4 were investigated at different time points in EPA treated ESCs. Our results showed that ID1 protein expression increased almost four fold after $3 \mathrm{~h}$ of EPA treatment with respect to the LIF treated ESCs used as control (Fig. 3E). In addition, we observed a significant decrease (88\%) of ID1 expression level after 24h of EPA treatment. On the other hand, ID2 proteins showed a slight decrease from $1 \mathrm{~h}$ up to $6 \mathrm{~h}$ after EPA treatment with respect to the control LIF ESCs (Fig. 3F). Interestingly, ID2 increased significantly (1.7 fold) over basal LIF level at $24 \mathrm{~h}$ following EPA treatment, suggesting a delayed ID2 activation. The expression of ID3 proteins after EPA treatment was similar to that of ID1 protein, reaching a peak at $3 \mathrm{~h}$ when compared to the LIF treated ESC levels (Fig. 3G). Finally, ID4 protein expression decreased progressively up to $24 \mathrm{~h}$ after EPA treatment of undifferentiated ESCs (Fig. 3H).

Altogether, our results show only modest changes in the expression levels of ID2 after EPA-treatment in the medulla, cerebellum and cortex of neonatal rats. In contrast to the brain tissues, EPA treatment of undifferentiated mESCs had a more pronounced effect on IDs expression levels. Probably in LIF-treated mESCs, EPA cooperate with LIF/STAT3 pathway to sustain mESC self-renewal through the induction of IDs (particularly ID1 and ID3 early expression), thus blocking neural lineage committment [51]. However, the upregulation of ID2, which is delayed with respect to ID1 and ID3, could be the consequence of a tight control of ID2 expression 
530 levels in LIF-treated mESCs. Indeed, elevated ID2 expression deregulates neural 531 stem cells self-renewal in mice [52]. In conclusion, EPA-treatment affects diversely 532 the ID protein expression levels in mESCs and pup rat brain, suggesting a G-protein 533 dependent or -independent mechanism for EPA signaling pathway.

3.5. CREBI and ID family members interact in the rat pup brain and in ESCs treated with EPA plus RA extracts were treated or not with 1h EPA and immunoprecipitated with anti-ID2 antibodies or normal rabbit serum as control. The resulting immunoprecipitates were immunoblotted using an antibody specific for the CREB1 protein (Fig. 4A).

A

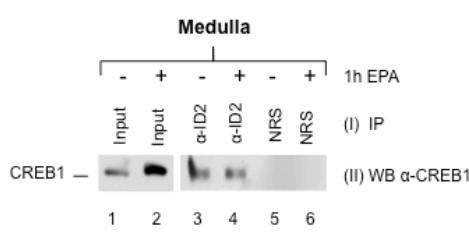

B

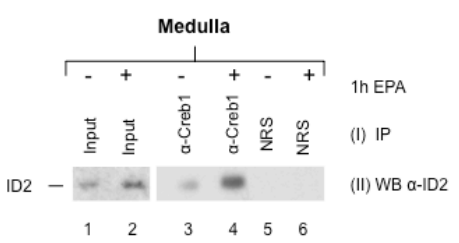

C

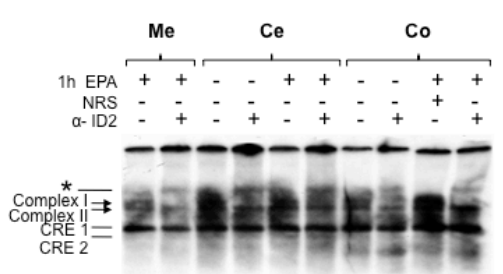

Ns -
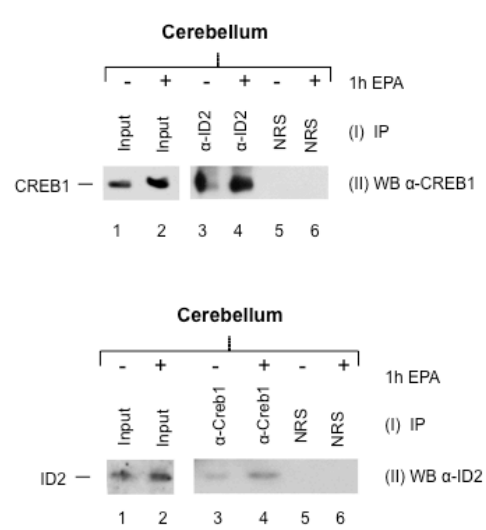

D

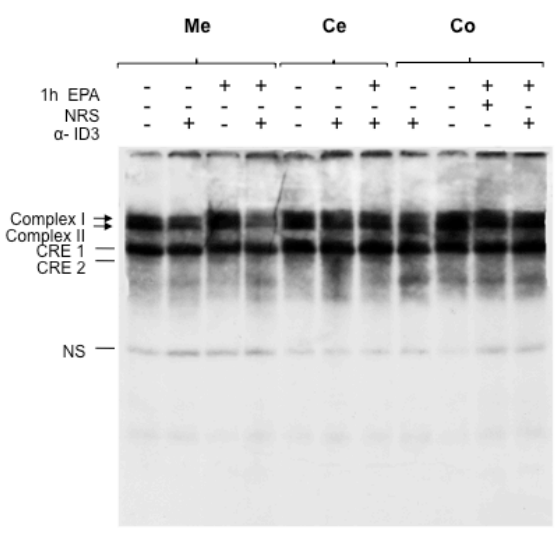

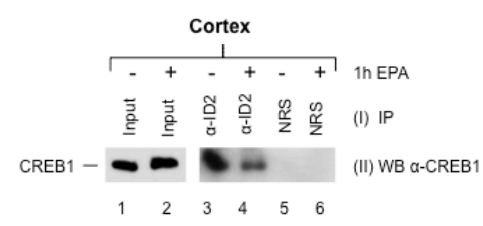

E

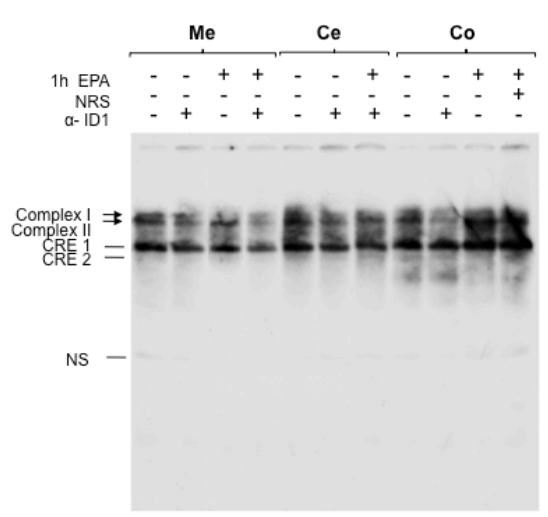


Fig. 4. CREB1 and ID family members interact in the rat pup brain. (A, B) CREB1 is complexed with ID2 protein in the medulla, cerebellum and cortex of the rat pup brain: reciprocal IP and WB analysis. Nuclear extracts from the medulla, cerebellum and cortex untreated or treated for $1 \mathrm{~h}$ with EPA were used as positive controls (lanes 1 and 2 in each panel). (C to E) EMSA analysis of CRE DNA-binding activity of nuclear extracts from medulla, cerebellum and cortex in rat pups treated with EPA at $1 \mathrm{~h}$ or untreated after incubation with an anti-ID2 serum (C, lanes 2,4,6,8,10), an anti-ID3 serum (D, lanes 2,4,6,7,8,11) and with an anti-ID1 serum (E, lanes 2,4,6,7,9). Supershifted complexes are marked by asterisks (C). NS, are nonspecific bands.

The CREB1 protein was immunoprecipitated in nuclear extracts from medulla, cerebellum and cortex with an anti-ID2 serum (Fig. 4A, lanes 3 and 4 in each panel) but not with the normal rabbit serum (Fig. 4A, lanes 5 and 6 in each panel). In the reciprocal experiments, nuclear extracts from the medulla, cerebellum, and cortex treated or untreated with 1h EPA were immunoprecipitated with anti-CREB1 antibodies, which co-precipitates only a single band, as revealed by western blot analysis with anti-ID2 antibody (Fig. 4B, lanes 3 and 4 in each panel). This band was absent in the normal rabbit serum immunoprecipitates (Fig. 4B, lanes 5 and 6 in each panel). Taken together, the coimmunoprecipitation and western blot experiments indicate a biochemical interaction between CREB1 and ID2 proteins, thus suggesting the presence of an ID2/CREB1 complex in the rat pup brain.

Based on our coimmunoprecipitation results, we performed supershift analysis with anti-ID2 antibody to elucidate the composition of the two high molecular weight CRE DNA-binding complexes in the rat pup brain. EMSA analysis of the CRE-DNA binding activity showed that only complex I was sharply supershifted when an antiID2 antibody was added to the reaction mixture of untreated or EPA treated medulla (Fig. 4C, lane 2), cerebellum (Fig. 4C, lanes 4 and 6), and cortex (Fig. 4C, lanes 8 and 10), but not with the normal rabbit serum used as control (Fig. 4C, lane 9). Therefore, a high molecular weight ID2:CRE-DNA protein complex is present in both untreated or 1h EPA treated brain extracts.

Subsequently, we investigated whether CRE-DNA binding complexes interact with other ID family proteins. EMSA analysis of CRE DNA-binding activity of 
nuclear extracts from rat pup medulla, cerebellum, and cortex untreated or treated with EPA for $1 \mathrm{~h}$ were incubated with anti-ID3 or anti-ID1-specific antiserum. We observed that the addition of an anti-ID3 antibody caused a decrease in the intensity of complex I and II, which was more evident in the medulla (Fig. 4 D, lanes 2 or 4) compared to the cerebellum and cortex (Fig. 4D, lanes 6, 7 and 8, 11 respectively). Similarly, the anti-ID1 antiserum caused a specific decrease in the intensity of the complex I and II in medulla (Fig. 4E, lanes 2 and 4), cerebellum (Fig. 4E, lanes 6 and 7), and cortex (Fig. 4E, lane 9). Taken together, these data indicate that ID2, and probably ID1 and ID3 proteins, can interact with high molecular weight CRE-DNA binding complexes in all pup brain regions.

Finally, we attempted to further define the protein components of the two high molecular weight CRE DNA-binding complexes that were reformed within $24 \mathrm{~h}$ after RA differentiation of ESCs. For this purpose, we performed supershift analysis with an anti-ID2 antibody in EPA plus 24h RA-treated ES cells at different time points. Similar to the EMSA experiments obtained with rat pup brain, we observed that complex I in EPA plus RA-treated ES cell, was sharply supershifted when an antiID2 antibody was added to the reaction mixture (Fig. 2E, lanes 3, 5 and 7) but not with the normal rabbit serum used as control (Fig. 2E, lane 8). It is remarkable that the intensity of the complex I supershift caused by the anti-ID2 antibody is related to the length of EPA treatment in the RA differentiating ESCs. We speculate that this dosage dependent kinetic could reflect the induction of EPA treatment in stimulating the binding of ID2 protein into the CRE-DNA binding complex I during early stages of neural commitment of $\mathrm{m}$ ESCs. Taking into account the presence of $\mathrm{p}$-CREB1 or t-CREB1 in the complex I of ESCs (Fig. 2B, lanes 3 and 4), these observations strongly suggest that an ID2:CREB1-DNA binding complex was efficiently reformed and up-modulated by EPA treatment during the early stages of RA induced ESC differentiation. 
3.6. E47 protein expression and CREB1:E2A interaction in RA differentiating ESCs and in the rat pup brain after EPA treatment

Although the E2A gene has been regarded as ubiquitously expressed [21], variations in E12 and E47 protein expression among different tissues has been observed [53]. To determine whether EPA affects E2A transcription factor activity, the level of E47 protein was investigated in ESCs treated with RA after LIF withdrawal. Interestingly, western blot analysis of nuclear extracts from RA differentiating ESCs showed an increase (2.8 fold) of E47 protein expression after 24 h of EPA treatment compared to the control LIF sample (Fig. 5A and B). This result is consistent with the hypothesis that EPA can modulate E2A proteins in differentiating ESCs. Subsequently, we investigated whether CRE DNA-binding complexes can directly interact with the E12/E47 protein members during RAinduced neural differentiation of mESCs. In our EMSA experiments, we found that the anti-E12 antiserum sharply decreased the intensity of complex I, complex II and complex CRE3 in nuclear extracts from ESCs treated with RA for 24h plus 3h EPA (Fig. 2D, lane 5), while they were not affected by normal rabbit serum used as control (Fig. 2D, lane 4). Therefore, the E12 antibody may affect the intrinsic DNA binding ability of complex I, complex II and complex CRE3 without causing a supershift. These results suggest that E2A plays a role in the early stages of neural induction of ESCs generating functionally varied E2A:CRE DNA-binding complexes. 


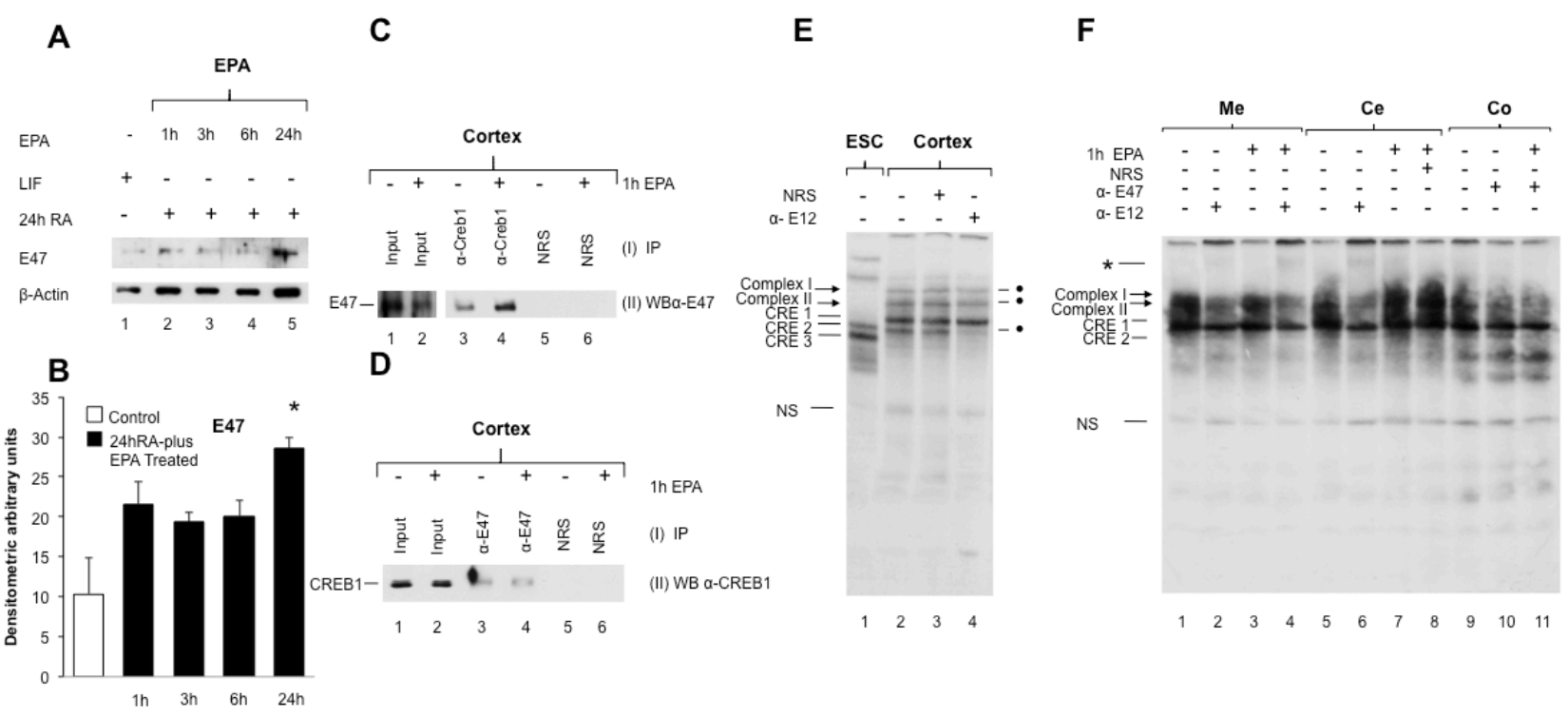

Fig. 5. E47 protein expression and CREB1:E2A interaction after EPA treatment. $(A, B)$ E47 protein expression in RA differentiating ESCs after EPA treatment at different time points. The levels of E47 protein were analysed by densitometric scanning and reported in the histogram after normalization to $\beta$-actin. Values are expressed as mean \pm SEM of three independent experiments. ${ }^{*} \mathrm{P}<0.05$ vs. control. (C, D) E47 is complexed with CREB1 protein in the cortex of the rat pup brain: reciprocal IP and WB analysis. (E) EMSA assay of CRE-DNA binding activity of nuclear extracts from cortex after incubation with an anti-E12 antibody. Extracts from ESCs treated with LIF were used as control (lane 1). Dots denote distinct bands that are decreased in signal intensity by the anti-E12 antibody (lane 4). (F) EMSA analysis of CRE DNA-binding activity of nuclear extracts from medulla, cerebellum and cortex in rat pups untreated (lanes $1,2,5,6,9,10$ ) or treated (lanes $3,4,7,8,11$ ) with EPA after incubation with anti-E12 or anti-E47 antibodies. Supershifted complexes are marked by asterisk (lanes $2,4,6$ ). NS, are nonspecific bands.

Furthermore, we investigated the "in vivo" interactions between CREB1 and E12/E47 proteins in the rat pup brain. Nuclear extracts from cortex were treated or untreated with EPA for $1 \mathrm{~h}$ and immunoprecipitated with anti-CREB1 antibodies or normal rabbit serum used as control. The resulting immunoprecipitates were immunoblotted using an antibody specific for E47 protein (Fig. 5C). The E47 protein was immunoprecipitated with anti-CREB1 antibodies (Fig. 5C, lanes 3 and 4) but not with the normal rabbit serum used as control (Fig. 5C, lanes 5 and 6). In the reciprocal immunoprecipitation experiments with anti-E47 antibodies (Fig. 5D), only a single immunoprecipitated band was detected by western blot analysis with antiCREB1 antibody (Fig. 5D, lanes 3 and 4). In conclusion, our coimmunoprecipitation experiments indicate the presence of an E2A:CREB1 complex in the rat pup brain. 
Finally, we performed supershift experiments with anti-E2A antibodies in the rat pup brain. Briefly, the anti-E12 antiserum caused a specific supershift of both complexes I and II in the medulla (Fig. 5F, lanes 2 and 4) and the cerebellum (Fig. $5 \mathrm{~F}$, lane 6). In addition, the anti-E12 and the anti-E47 antiserum significantly and specifically decreased the bands related to complex I and complex II in the cortex (Fig. 5E and 5F). Interestingly, the E12 antibody induced a decrease of the CRE2 band in the cortex, where this isoform is expressed (Fig. 5E, lane 4). Taken together, our data suggest that E12 and E47 can interact with different CRE DNA-binding complex in specific region of the rat pup brain to form stable dimeric (CREB:E2A), trimeric (CREB:ID:E2A) or tetrameric ([CREB $\left.]_{2}: \mathrm{ID}: \mathrm{E} 2 \mathrm{~A}\right)$ naturally assembled complexes on a CRE consensus site.

\subsection{Structural model of the [CREB1] $2: I D 2: E 47$ tetramer bound to the CRE site of} $D N A$

Focusing on our hypothesis that ID2, E47 and CREB1 are found in a single complex when bound to the CRE-site (see experiments above), we aimed to build a structural model for this complex. This model is based on the CREB1 basic leucine zipper domain and the HLH domain of ID2 and E47 (as described in Materials and Methods). Crystal structures of the corresponding domains have been solved [32, 54, 55]. The stoichiometry and the 3D structural arrangement of the domains in the tetrameric complex are, however, elusive. The helix-loop-helix motifs of ID2 and E47 are known to form homo- and hetero-dimers $[32,54]$. EMSA experiments performed using purified E47 and ID2 proteins showed a dose response effect, which is indicative of a direct interaction between these two proteins [32]. Under physiological conditions ID2 represses E47 by forming heterodimers [32, 56]. Therefore, we favor a model with E47:ID2 heterodimer. CREB1 homodimerizes 
672 when bound to the CRE-site of DNA [55], but it can also form heterodimers by direct 673 interaction with bHLH proteins as suggested by Skinner and coworkers [57]. From in 674 vitro binding assays, a recent report [30] suggested that CREB homodimer can be 675 stabilized in a tetrameric complex. Therefore, we propose that CREB1 homodimer 676 (bound to the CRE-site of DNA) interacts with E47:ID2 heterodimer. An indication 677 of how these two dimeric units could be arranged in a tetrameric structure comes 678 from the crystal structure of the tetramer of [E47: NeuroD1 $]_{2}$ (see Fig. 6A, right) 679 680 681 682 683 684 685 686 687 688 689 690 691 $6926 \mathrm{C})$. [54]. Using this tetramer as template, we obtained an initial model through superimposition with other crystal structures (see Material and Methods). First, NeuroD1 was substituted by ID2 in one of the two NeuroD1:E47 units. NeuroD1 and ID2 feature a very similar three-dimensional HLH arrangement so that the NeuroD1ID2 exchange leads to a matching interface with E47. Second, by replacing the other NeuroD1:E47 unit with a CREB1 homodimer (Fig. 6A, left) we obtained a multimer that showed a complementary contact surface between the CREB1 homodimer and the ID2:E47 unit with an interface area of $922 \AA^{2}$. There was, however, a small cleft between these two dimers. Slight rotation (see Material and Methods) of the CREB1 homodimer and the ID2:E47 unit relative to each other (as indicated in Fig. 6A) maximizes the size of the interface $\left(1012 \AA^{2}\right)$ and leads to the structure shown in Fig. 6B. The structural model is available upon request. A short molecular dynamics 691 simulation (500 ns) indicates that this structure is a (meta)-stable conformation (Fig. 


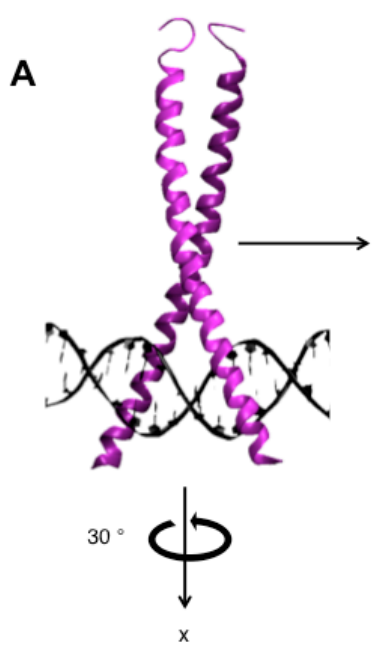

C

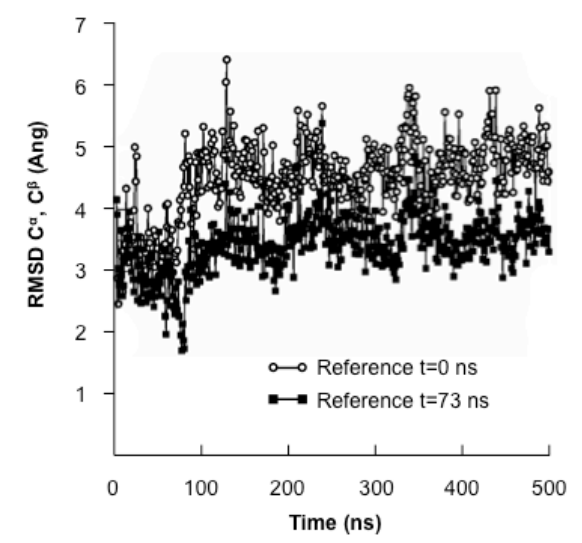

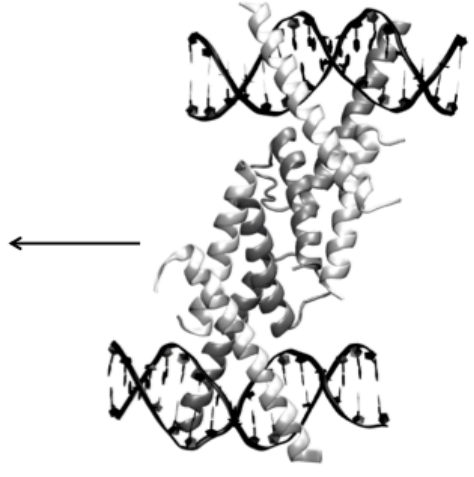

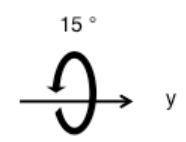

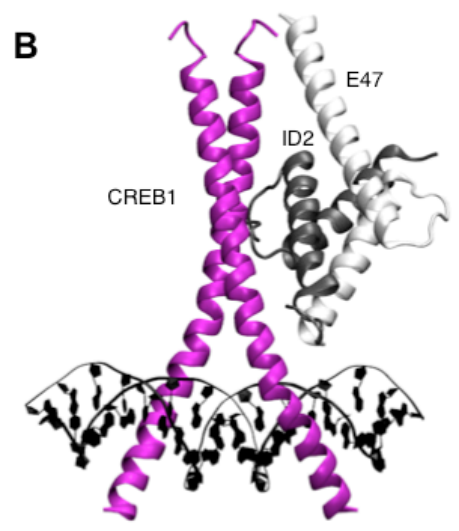
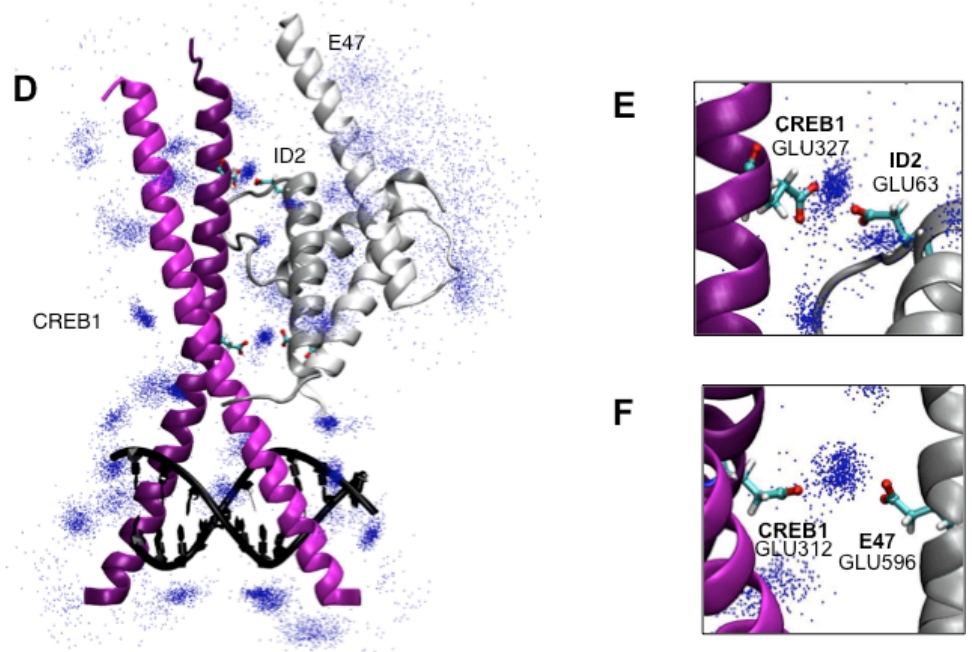

Fig. 6. Structural model and molecular dynamics of the [CREB1] $]_{2}$ ID2:E47 tetramer bound to DNA. (A) Crystal structure of the CREB dimer (left, PDB ID: 1DH3), and tetramer structure of NeuroD1 (dark grey) and E47 (light grey) (right, 2QL2). (B) It is possible to overlay the CREB dimer on one of the NeuroD1:E47 units and thereby generate a matching binding interface with the other NeuroD1:E47 unit. Replacing NeuroD1 by ID2 yields the structure displayed in the figure. (C) The root-mean-square deviation of the $\mathrm{C}^{\alpha}$, $\mathrm{C}^{\beta}$-atoms is shown for the complex structure of figure $6 \mathrm{~B}$ during a molecular dynamics trajectory of $500 \mathrm{~ns}$ at temperature of $310 \mathrm{~K}$. Using the initial structure as reference ( $\mathrm{t}=0 \mathrm{~ns}$, open circles), the formation of a plateau is observed at about $70 \mathrm{~ns}$. The remaining trajectory shows a constant and lower RMSD with respect to a reference structure picked at $73 \mathrm{~ns}$ (filled squares). (D) Snapshot structure picked at $73 \mathrm{~ns}$ of the molecular dynamics trajectory. (For reasons of clarity, terminal and highly flexible nucleotides are not shown). An overlay of all calcium positions sampled during the trajectory reveals the most likely calcium binding sites (blue cloud). (E, F) Two sites are found at the interface of ID2 and CREB1where glutamic acids from both monomers contribute to the binding. 
710 In the model of Fig. 6B the ID2 subunit is the primary interactor with the CREB1 711 homodimer. In principle, one can hypothesize an alternative arrangement, where the 712 E47 unit faces the CREB1 dimer. Docking studies (see Material and Methods) 713 indicate, however, that the CREB1 homodimer preferentially interacts with the ID2 714 subunit: the mean contribution of ID2 to the buried surface area (when averaged over 715 the ten top-ranked docking predictions) is $734 \pm 157 \AA^{2}$. The contribution of E47 is 716 three times smaller $\left(230 \pm 134 \AA^{2}\right)$. In the model of Fig. $6 \mathrm{~B}$ these contributions are 717673 and $309 \AA^{2}$ for ID2 and E47, respectively, in agreement with the docking results. 718 Therefore, the alternative binding mode where E47 faces the CREB1 homodimer was 719 not taken into further consideration. It has been proposed that rigidification of the ID2 loop is necessary for heterodimer formation (e.g., with E47) [32]. In our tetrameric model, the ID2 loop (residues 4863) forms multiple intermolecular hydrogen bonds with CREB1. Thus, binding of ID2 to $[\mathrm{CREB} 1]_{2}$ confines the ID2 loop into a specific, rigid conformation that may favour the ID2:E47 heterodimer formation.

Distinct calcium $\left(\mathrm{Ca}^{2+}\right)$-binding sites can be identified for the [CREB1 $]_{2}$ : ID2: E47 tetramer (Fig. 6D). Remarkably, the most pronounced sites are found in the binding interface between ID2:E47 and [CREB1] $]_{2}$. Glutamic acids from ID2 (Glu63), E47 (Glu596) and CREB1 (Glu312; Glu327) contribute to the binding of $\mathrm{Ca}^{2+}$ (Fig. 6E and F). There is another $\mathrm{Ca}^{2+}$-binding site close to the ID2-loop (Glu48). When bound to Glu48 the calcium ion also weakly interacts $(5 \%$ of sampled molecular dynamics-snapshots) with the side chain of the central residue of the ID2-loop (Gln55) (Supplementary Fig. 2A). This may further rigidify the ID2-loop. This observation supports previous results that $\mathrm{Ca}^{2+}$-binding to the ID2- loop is important for heterodimer formation [32].

735 Furthermore, the simulation revealed several $\mathrm{Ca}^{2+}$-binding sites involving acid amino 736 acids of CREB1 (e.g., Glu292 and Glu295) and phosphate groups of the DNA, 737 (Supplementary Fig. 2B). It is known that divalent cations (i.e. $\mathrm{Ca}^{2+} ; \mathrm{Mg}^{2+}$ ) enhance 
significantly (more than 25 times) the binding of CREB1 to DNA [55]. Thus, the stability of the proposed tetrameric protein complex and its binding to the DNA is potentially influenced by the $\mathrm{Ca}^{2+}$ concentration. For visualization purposes an additional movie (Video $\mathrm{S} 1$ ) of the identified $\mathrm{Ca}^{2+}$ binding sites in the tetrameric complex, is included in the supplementary materials.

\section{DISCUSSION}

\subsection{EPA modulates the interaction between the HLH:bHLH proteins ID and E2A} and the CREB/ATF family member CREBI

The purpose of this study was to elucidate the molecular mechanism by which EPA may exert its beneficial effects in neurological diseases. The current study reveals that EPA modulates the exquisite interplay of interaction of the HLH:bHLH proteins ID and E2A and the CREB/ATF family member CREB1, thereby delivering mechanistic insights into specific neural differentiation program. Due to the importance of HLH:bHLH and CREB1 transcription factors in key developmental events, this association may be at the basis of general mechanisms that regulate multiprotein complex formation in other differentiation processes.

Here, we show the ability of EPA to up-modulate p-CREB1 at early times of treatment in ESCs and to induce the CREB1 occupancy at a non-canonical CRE site in the myelin PLP promoter. These data suggest that an EPA-CREB signaling pathway activates PLP transcription. However, p-CREB1 alone is not sufficient to activate target gene transcription. To clarify this regulatory aspect, EPA treatment might provide a novel level of specificity of CREB1 target gene activation by stabilizing the CREB1:HLH:bHLH complex formation within the promoter region of distinct glial or neural genes involved in the coordination of myelin biogenesis. This protein complex assembly was suggested by our coimmunoprecipitation and EMSA 
analysis of rat pup brain and RA differentiating ESCs after EPA treatment, together with our previous data on the expression of myelin proteins [15]. More specifically, we provide evidence for the natural assembly of CREB1, ID2, and E47 on a CRE consensus site. On the basis of the crystal structures of the CREB basic leucine zipper-CRE complex [55], ID2 homodimers [32] and the E47/ Neuro D1 [54], we could establish a structural model for this assembly (with a 2:1:1 stoichiometry of CREB1, ID2 and E47) that yields a stable molecular dynamics trajectory.

\subsection{Biological relevance of the interaction between CREB1 and ID2}

An important aspect for examining the interaction between CREB1 and ID2 is related to the key role played by ID2 in neurodevelopmental disorders that raised our interest in the investigation of the EPA-CREB-ID2 axis. Even though ID1 and ID3 appear more dynamically regulated than ID2 in mESCs after EPA treatment, only the ID2-antibody yielded a distinct supershift of the multimer formed by ID2 with CREB1 (Fig. 2E). Indeed, ID2 inhibits oligodendroglia differentiation [58] which is required for remyelination, and might have a role in multiple sclerosis, a disease characterized by axonal demyelination $[59,60]$.

We noted that medulla, cerebellum and cortex of neonatal rats show only very modest changes in expression levels of ID2 after EPA-treatment. In contrast in LIFtreated mouse ESCs, EPA-treatment displays a much more pronounced effect on ID protein levels; the upregulation of ID2 is, however, delayed with respect to ID1 and ID3. In the same way, in the rat pup brain, we observed no effect at $1 \mathrm{~h}$ of EPA treatment in the profile of CRE-DNA binding activity and particularly of Complex I containing CREB1 and ID2 proteins. In contrast, in the EPA treated mESCs after 24 $\mathrm{h}$ of RA-induced differentiation, both complexes I and II were up-modulated according to the time of EPA treatment, reaching the highest intensity at the end of the experiments (6h after treatment, Fig. 2E). This time-dependent response can be seen as an indirect evidence for EPA-induced assembly of ID2 and CREB1 into the 
high-molecular weight complex I during early stages of neural commitment of mESCs.

\subsection{How we can explain this evidence regarding the difference in EPA treatment} between mouse and rat?

In this respect, recent studies underline the complexity of the mechanism regulating gene expression by dietary $\omega-3$ PUFA $[16,61]$. The effect of EPA in neurogenesis may need the availability of cell surface $\omega-3$ PUFA receptors, specific fatty acid binding proteins and other regulatory trascriptional proteins (including PPARs, RXR and RA receptors) that are crucial for the induction of the signal trasduction processes. Recent data suggest that GPR40 (also known as FFA1/FFAR1), a seven-transmembrane domain receptor, can bind PUFA to induce $\mathrm{Ca}^{2+}$ mobilization which phosphorylates CREB1 promoting CREB-mediated gene transcription in neural cells $[61,63]$. Expression of GPR40 has been confirmed in various areas of the monkey and human central nervous system [63]. Interestingly, GPR40 induced pCREB1 by activating a mitogen activated protein kinase/extracellular signal-regulated kinase cascade for neural differentiation and synaptic plasticity [16]. Therefore, PUFA, GPR40 and pCREB1 appear involved in the same signaling pathways promoting adult neurogenesis in primates [64].

According to previously reported data [16], the rat pup brain lacks the cell surface GPR40 receptor and this might cause a temporal delay of EPA effects ( $>1 \mathrm{~h})$. Our previous results in the rat pup brain showed that EPA stimulates the expression of myelin PLP and myelin oligodendrocyte glycoprotein transcripts from day 1 postinjection in the medulla and cerebellum, whereas in the cortex EPA effects on PLP and oligodendrocyte glycoprotein transcripts are evident only at day 3 post-injection [15]. Indeed, a significant expression of GPR40 in primary culture of mouse neurons [65] and brain [66] were recently reported. Probably, the GPR40 receptor on the extracellular membrane of mESCs favoured the conduction of EPA induced signals 
821 to the nucleus for CREB1 phosphorylation at early time points. In addition, RA822 induced differentiation of mESC may involve PPAR/RXR nuclear receptor complex 823 that triggers the EPA-GPR40-CREB signaling pathway. Thus, the mouse may 824 represent a more reliable model to address the role of the PUFA-GPR40-CREB 825 signaling pathway during early neural development in humans. More importantly, the 826

\subsection{CREB1 sequestration model of ID2-E47 heterodimers after EPA treatment in} murine neural cell differentiation.

From our analysis, the molecular function of CREB1 can be to dynamically regulate the location-specific assembly or disassembly of bHLH:HLH proteins complex on neural/glial target genes. CREB1 monomers bind DNA sequentially to CRE-binding sequence according to an assembly pathway as previously described [67]. During neural specification, CREB1 homodimers sequester inactive (non-DNA binding) ID:E2A heterodimers in an active CRE DNA-binding complex (Fig. 7). Therefore, in our CREB1 sequestration model, DNA-bound CREB1 homodimers relocate ID2:E47 containing complexes and fine-tune their activities through potential interaction with associated cofactors/chromatin remodelers (Fig. 7). 

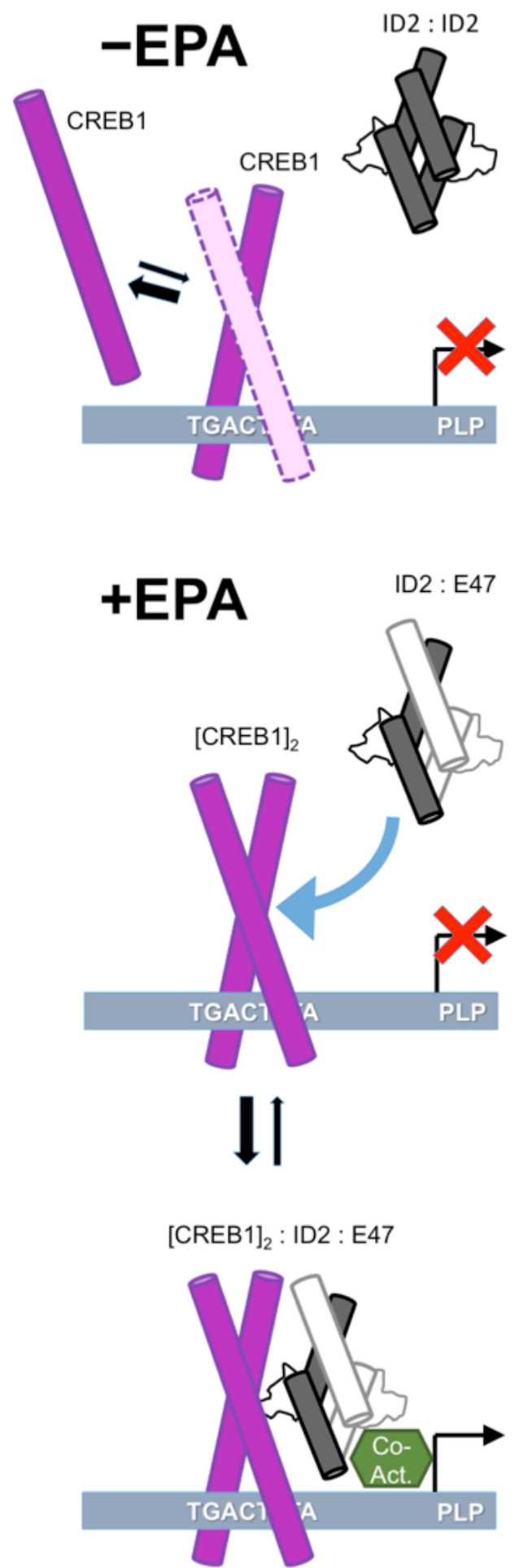

843 Fig. 7. CREB1 sequestration model of ID2-E47 heterodimers after EPA treatment in murine neural cell 844 differentiation. CREB1 monomers bind DNA sequentially to the non-canonical CRE-binding sequence (5'845 TGACTTTA-3') present in the mouse myelin PLP promoter according to an assembly pathway as previously 846 described [67]. ID2 exists as homodimers in its native state [32]. After EPA addition, the increase of $\mathrm{Ca}^{2+}$ 847 favours the ID2-E47 heterodimerization [32] and enhances CREB1 dimerization and DNA-binding affinity 848 to CRE box sequence carrying degenerated semi-palindrome on the PLP promoter [55]. During neural 849 specification, CREB1 homodimers sequester ID2:E47 heterodimers (this work) generating an active 
850

851

852

853

854

855

856

857

858

859

860

861

862

863

864

865

866

867

868

869

870

871

872

873

874

875

[CREB1] $2:$ ID2:E47 tetrameric complex that initiate transcription through potential interactions with associated cofactors. See Discussion for details.

The proposed sequestration model supports the one-to-many binding mechanism of CREB1, whereby CREB- sequestering proteins and associated cofactors/chromatin remodelers (i.e. the repressor element-1 silencing transcription factor (REST) and the corepressor CoREST) can regulate neural lineage specific CREB1-target genes [68]. In addition, E47 binds the histone methyltransferase LSD1 to activate neural genes containing CRE sites [69]. This model can also explain how a limited number of $\mathrm{E}$ proteins binding to CREB1 homodimers generates functionally varied complexes conferring diversity to neural cells.

Our structural tetrameric model provide evidence that $\mathrm{Ca}^{2+}$ can modify the CRE DNA-binding affinity of the complex I increasing the residence time of the [CREB1]2:ID2:E47 tetrameric complex on DNA. In so doing, a CRE box sequence carrying a degenerated semi-palindrome (Fig. 7) may be sufficient for specific genomic target recognition [30]. Therefore, an additional level of promoters/enhancers specific binding is achieved by the [CREB1] $]_{2}:$ ID2:E47 tetrameric complex, having physiological relevance. Furthermore, ID2:E47 heterodimer stability is also influenced by $\mathrm{Ca}^{2+}$ [32], thus variation in $\mathrm{Ca}^{2+}$ levels may allow the release of ID2 and the E47 from the tetrameric complex (Fig. 6E and F). Subsequently, ID2 and E47 proteins into different DNA-bound complexes or sequestering cofactors can activate or repress specific target genes thereby triggering a cascade of regulatory events controlled by CREB1. 
Consistent with our findings, an interaction between CREB family members and bHLH has been reported in Sertoli cells and myogenic precursors where ATF4/CREB2 and sclerasis heterodimerize, thus integrating the transcriptional signaling pathways [57]. Interestingly, a CREB1: Lyl-1 complex is present in acute myeloid leukemia and alters the expression of CREB1 target genes [70]. Furthermore, a CREB1: Lyl-1 complex is assembled on the Stathmin promoter at a CRE site [71]. Therefore, similar models of HLH:bHLH DNA-binding specificity driven by CREB/ATF family members are emerging in spermatogenesis, myogenesis leukemiogenesis and neurogenesis. Thus, HLH:bHLH bound protein partners are directed to lineage specific CREB target genes. Importantly, integrative genomic analysis of CREB [72] shows that transcription factors from multiple families (i.e. PPAR-alpha, glucocorticoid receptor (NR3C1), CCAAT/enhancer-binding protein beta and forkhead box protein A2) influence the direction of the CREB regulatory response to the feeding/ fasting metabolic switch in the liver, suggesting a more widespread cooperation between CREB1 and transcriptional regulators on individual regulatory elements at CREB target genes.

\section{CONCLUSIONS}

Overall, our results strongly support a role of EPA as an attractive molecule for the prevention of neurodegenerative diseases. The ability of CREB [73] and ID proteins [74-76] to function as central "hubs" for the coordination of multiple pathways controlling the timing of neural stem cell differentiation during development may share light on the symbiotic relationship of the axon and myelin sheath in the central nervous system. As strongly suggested by recent findings [77], this interaction could play a key role in the pathogenesis of multiple sclerosis. Future studies on the EPA-CREB axis should reveal new molecular targets for multiple sclerosis treatment. 
We are very gratefull to Stefano Fortuna from the Department of Therapeutic Research and Medicines Evaluation, for technical support and to Massimo Delle Femmine for the preparation of the figures. This research was supported by institutional grants from the Italian Ministry of Health to L.V. and C.N. M.S. is grateful to the Centre Blaise Pascal and the Pôle Scientifique de Modélisation Numérique (both ENS Lyon) for their support on GPU-based calculations.

L.V. supervised the project, performed cell culture of embryonic stem cells, and wrote the paper. M.R., L.A. and C.D. performed biochemical experiments and data analysis. M.S. performed the molecular dinamics simulations. S.S. and C.N. provided essential reagents and intellectual support.

We declare that we have no conflicts of interest with the contents of this article.

\section{Appedix A. Supplementary data}

\section{REFERENCES}

[1] J.D. Figueroa, K. Cordero, K. Baldeosingh, A.I. Torrado, R.L. Walker, J.D. Miranda, M.D. Leon, Docosahexaenoic acid pretreatment confers protection and functional improvements after acute spinal cord injury in adult rats, J. Neurotrauma 29 (2012) 551-566, http://dx.doi.org/10.1089/neu.2011.2141.

[2] S. Salvati, A. Di Biase, L. Attorri, R. Di Benedetto, M. Sanchez, L. Lorenzini, M. Alessandri, L. Calzà, Ethyl-eicosapentaenoic acid ameliorates the clinical course of experimental allergic encephalomyelitis induced in dark agouti rats, J. Nutr. Biochem. 24 (2013) 1645-1654, http://dx.doi.org/10.1016/j.jnutbio.2013.02.005.

[3] A. Di Biase, S. Salvati, R. Di Benedetto, L. Attorri, M. Martinelli, F. Malchiodi, Eicosapentaenoic acid pretreatment reduces biochemical changes induced in total brain and myelin of weanling Wistar rats by cuprizone feeding, Prostaglandins Leukot. Essent. Fatty Acids 90 (2014) 99-104,

http://dx.doi.org/10.1016/j.plefa.2013.11.004.

[4] S.C. Dyall, A.T. Michael-Titus, Neurological benefits of omega-3 fatty acids, 
936

[5] X. Yang, W. Sheng, G.Y. Sun, J.C.M. Lee, Effects of fatty acid unsaturation numbers on membrane fluidity and $\alpha$-secretase-dependent amyloid precursor protein processing, Neurochem. Int. 58 (2011) 321-329,

http://dx.doi.org/10.1016/j.neuint.2010.12.004.

[6] S. Hong, K. Gronert, P.R. Devchand, R.L. Moussignac, C.N. Serhan, Novel docosatrienes and 17S-resolvins generated from docosahexaenoic acid in brain, human blood, and glial cells. Autacoids in anti-inflammation, J. Biol. Chem. 278 (2003) 14677-14687, http://dx.doi.org/10.1074/jbc.M300218200.

[7] B.H. Maskrey, I.L. Megson, A.G. Rossi, P.D. Whitfield, Emerging importance of omega-3 fatty acids in the innate immune response: Molecular mechanisms and lipidomic strategies for their analysis, Mol. Nutr. Food Res. 57 (2013) 1390-1400, http://dx.doi.org/10.1002/mnfr.201200723.

[8] S.D. Clarke, The multi-dimensional regulation of gene expression by fatty acids: polyunsaturated fats as nutrient sensors, Curr. Opin. Lipidol. 15 (2004) 13-18.

[9] S. Lorente-Cebrián, M. Bustos, A. Marti, J.A. Martinez, M.J. Moreno-Aliaga, Eicosapentaenoic acid up-regulates apelin secretion and gene expression in 3T3-L1 adipocytes, Mol. Nutr. Food Res. 54 (2010) S104-S111, http://dx.doi.org/10.1002/mnfr.200900522.

[10] V. Ceccarelli, G. Nocentini, M. Billi, S. Racanicchi, C. Riccardi, R. Roberti, F. Grignani, L. Binaglia, A. Vecchini, Eicosapentaenoic Acid Activates RAS/ERK/C/EBPß Pathway through H-Ras Intron $1 \mathrm{CpG}$ Island Demethylation in U937 Leukemia Cells, PLoS ONE 9 (2014) e85025, http://dx.doi.org/10.1371/journal.pone.0085025.

[11] A.M. de Urquiza, S. Liu, M. Sjöberg, R.H. Zetterström, W. Griffiths, J. Sjövall, T. Perlmann, Docosahexaenoic Acid, a Ligand for the Retinoid X Receptor in Mouse Brain, Science 290 (2000) 2140-2144, http://dx.doi.org/10.1126/science.290.5499.2140.

[12] J. Lengqvist, A. Mata De Urquiza, A.C. Bergman, T.M. Willson, J. Sjövall, T. Perlmann, W.J. Griffiths, Polyunsaturated fatty acids including docosahexaenoic and arachidonic acid bind to the retinoid $\mathrm{X}$ receptor alpha ligand-binding domain, Mol. Cell Proteomics, 3 (2004) 692-703, http://dx.doi.org/10.1074/mcp.M400003MCP200. 
[13] K. Unoda, Y. Doi, H. Nakajima, K. Yamane, T. Hosokawa, S. Ishida, F. Kimura, T. Hanafusa, Eicosapentaenoic acid (EPA) induces peroxisome proliferator-activated receptors and ameliorates experimental autoimmune encephalomyelitis, Journal of

971 Neuroimmunology, 256 (2013) 7-12,

972 http://dx.doi.org/10.1016/j.jneuroim.2012.12.003.

973

974

975

976

977

978

979

980

981

982

983

984

985

986

987

988

989

990

991

992

993

994

995

996

997

998

999

000

.001

[14] S. Salvati, F. Natali, L. Attorri, C. Raggi, A. Di Biase, M. Sanchez, Stimulation of myelin proteolipid protein gene expression by eicosapentaenoic acid in C6 glioma cells, Neurochem. Int. 44 (2004) 331-338, http://dx.doi.org/10.1016/S01970186(03)00172-4.

[15] S. Salvati, F. Natali, L. Attorri, R. Di Benedetto, F. Leonardi, A. Di Biase, F. Ferri, S. Fortuna, P. Lorenzini, M. Sanchez,L. Ricceri, L. Vitelli, Eicosapentaenoic acid stimulates the expression of myelin proteins in rat brain, J. Neurosci. Res. 86 (2008) 776-784, http://dx.doi.org/10.1002/jnr.21537

[16] T. Yamashima, PUFA-GPR40-CREB signaling hypothesis for the adult primate neurogenesis. Prog. Lipid Res. 51 (2012) 221-231, http://dx.doi.org/10.1016/j.plipres.2012.02.001.

[17] B.E. Lonze, D.D. Ginty, Function and regulation of CREB family transcription factors in the nervous system, Neuron 35 (2002) 605-623, http://dx.doi.org/10.1016/S0896-6273(02)00828-0

[18] X. Zhang, D.T. Odom, S.H. Koo, M.D. Conkright, G. Canettieri, J. Best, H. Chen, R. Jenner, E. Herbolsheimer, E. Jacobsen, S. Kadam, J.R. Ecker, B. Emerson, J.B. Hogenesch, T. Unterman, R.A. Young, M. Montminy, Genome-wide analysis of cAMP-response element binding protein occupancy, phosphorylation, and target gene activation in human tissues, Proc. Natl. Acad. Sci. USA 102 (2005) 4459-4464, http://dx.doi.org/10.1073/pnas.0501076102.

[19] J.Y. Altarejos, M. Montminy, CREB and the CRTC co-activators: sensors for hormonal and metabolic signals. Nat. Rev. Mol. Cell Biol. 12 (2011) 141-151, http://dx.doi.org/10.1038/nrm3072.

[20] M. Katakura, M. Hashimoto, T. Okui, H.M. Shahdat, K. Matsuzaki, O. Shido, Omega-3 polyunsaturated Fatty acids enhance neuronal differentiation in cultured rat neural stem cells. Stem Cells Int. 2013 (2013) 490476, http://dx.doi.org/10.1155/2013/490476.

[21] M.E. Massari, C. Murre, Helix-loop-helix proteins: regulators of transcription in eucaryotic organisms, Mol. Cell. Biol. 20 (2000) 429-440, 
[22] S. Jung, R.H. Park, S. Kim, Y.J. Jeon, D.S. Ham, M.Y. Jung, S.S. Kim, Y.D. Lee, C.H. Park, H. Suh-Kim, Id proteins facilitate self-renewal and proliferation of neural stem cells, Stem Cells Dev. 19 (2010) 831-841, http://dx.doi.org/10.1089/scd.2009.0093.

[23] F. Niola, X. Zhao, D. Singh, A. Castano, R. Sullivan, M. Lauria, H.S. Nam, Y. Zhuang, R. Benezra, D. Di Bernardo, A. Iavarone, A. Lasorella, Id proteins synchronize stemness and anchorage to the niche of neural stem cells, Nat. Cell Biol. 14 (2012) 477-487, http://dx.doi.org/10.1038/ncb2490.

[24] B. Fischer, K. Azim, A. Hurtado-Chong, S. Ramelli, M. Fernández, O. Raineteau, E-proteins orchestrate the progression of neural stem cell differentiation in 8104-9-23.

[25] T.J. Haley, W.G. McCormick, Pharmacological effects produced by intracerebral injection of drugs in the conscious mouse, Br. J. Pharmacol. 12 (1957) $12-15$.

[26] P. Savatier, H. Lapillonne, L. Jirmanova, L. Vitelli, J. Samarut, Analysis of the Cell Cycle in Mouse Embryonic Stem Cells, Methods Mol. Biol. 185 (2002) 27-33.

[27] L. Vitelli, G. Condorelli, V. Lulli, T. Hoang, L. Luchetti, C.M. Croce, C. Peschle, A pentamer transcriptional complex including tal-1 and retinoblastoma protein downmodulates c-kit expression in normal erythroblasts, Mol. Cell. Biol. 20 (2000) 5330-5342, http://dx.doi.org/10.1128/MCB.20.14.5330-5342.2000.

[28] J.J. Barrow, J. Masannat, J. Bungert, Neutralizing the function of ß-globinassociated cis-regulatory DNA element using an artificial zinc finger DNA-binding domain, Proc. Natl. Acad. Sci. U S A 109 (2012) 17948-17953, http://dx.doi.org/10.1073/pnas.1207677109.

[29] G. Condorelli, L. Vitelli, M. Valtieri, I. Marta, E. Montesoro,V. Lulli, R. Baer, C. Peschle, Coordinate expression and developmental role of Id 2 protein and TAL1/E2A heterodimer in erythroid progenitor differentiation, Blood 86 (1995) 164175.

[30] Q. Luo, K. Viste, J.C. Urday-Zaa, G. Senthil Kumar, W.W.Tsai, A. Talai, K.E. Mayo, M. Montminy, I. Radhakrishnan, Mechanism of CREB recognition and coactivation by the CREB-regulated transcriptional coactivator CRTC2, Proc. Natl. 
Acad. Sci. U S A 109 (2012) 20865-20870, http://dx.doi.org/10.1073/pnas.1219028109.

[31] S. Pesce, R. Benezra, The loop region of the helix-loop-helix protein Id1 is critical for its dominant negative activity, Mol. Cell. Biol. 13 (1993) 7874-7880, http://dx.doi.org/10.1128/MCB.13.12.7874.

[32] M.V. Wong, S. Jiang, P. Palasingam, P.R. Kolatkar, A divalent ion is crucial in the structure and dominant-negative function of ID proteins, a class of helix-loophelix transcription regulators, PLoS One 7 (2012) e48591, http://dx.doi.org/10.1371/journal.pone.0048591.

[33] B.R. Brooks, C.L.3rd Brooks, A.D. Jr. Mackerell, L. Nilsson, R. J. Petrella, B. Roux, Y. Won, G. Archontis, C. Bartels, S. Boresch, A. Caflisch, L. Caves, Q. Cui, A.R. Dinner, M. Feig, S. Fischer, J. Gao, M. Hodoscek, W. Im, K. Kuczera, T. Lazaridis, J. Ma, V. Ovchinnikov, E. Paci, R.W. Pastor, C.B. Post, J.Z. Pu, M. Schaefer, B. Tidor, R.M. Venable, H.L. Woodcock, X. Wu, W. Yang, D.M. York, M. Karplus, CHARMM: the biomolecular simulation program, J. Comput. Chem. 30 (2009) 1545-614, http://dx.doi.org/10.1002/jcc.21287.

[34] S. Jo, T. Kim, V.G. Iyer, W. Im, CHARMM-GUI: a web-based graphical user interface for CHARMM, J. Comput. Chem. 29 (2008) 1859-1865, http://dx.doi.org/10.1002/jcc.20945.

[35] M.J. Sippl, Calculation of conformational ensembles from potentials of mean force. An approach to the knowledge-based prediction of local structures in globular proteins, J. Mol. Biol. 213 (1990) 859-883.

[36] F. Melo, E. Feytmans, Novel knowledge-based mean force potential at atomic level, J. Mol. Biol. 267 (1997) 207-222, http://dx.doi.org/10.1006/jmbi.1996.0868.

[37] B.G. Pierce, K. Wiehe, H. Hwang, B.H. Kim, T. Vreven, Z. Weng, ZDOCK server: interactive docking prediction of protein-protein complexes and symmetric multimers, Bioinformatics 30 (2014) 1771-1773, http://dx.doi.org/10.1093/bioinformatics/btu097.

[38] R.B. Best, X. Zhu, J. Shim, P.E. Lopes, J. Mittal, M. Feig, A.D.Jr. Mackerell, Optimization of the additive CHARMM all-atom protein force field targeting improved sampling of the backbone $\varphi, \psi$ and side-chain $\chi(1)$ and $\chi(2)$ dihedral angles, J. Chem. Theory Comput. 8 (2012) 3257-3273, http://dx.doi.org/10.1021/ct300400x.

[39] M.J. Harvey, G. Giupponi, G. De Fabritiis, ACEMD: Accelerating Biomolecular 
Dynamics in the Microsecond Time Scale, J. Chem. Theory Comput. 5 (2009) 1632-

[40] J.X. Kang, J.B. Wan, C. He, Concise review: Regulation of stem cell proliferation and differentiation by essential fatty acids and their metabolites, Stem 1639, http://dx.doi.org/10.1021/ct9000685. Cells 32 (2014) 1092-1098, http://dx.doi.org/10.1002/stem.1620.

[41] O. Yanes, J. Clark, D.M. Wong, G.J. Patti, A. Sánchez-Ruiz, H.P. Benton, S.A. Trauger, C. Desponts, S. Ding, G. Siuzdak, Metabolic oxidation regulates embryonic stem cell differentiation, Nat. Chem. Biol. 6 (2010) 411-417, http://dx.doi.org/10.1038/nchembio.364.

[42] P. Shabani, Z. Ghazizadeh, S. Pahlavan, S. Hashemizadeh, H. Baharvand, N. Aghdami, M. Doosti, Exogenous treatment with eicosapentaenoic acid supports maturation of cardiomyocytes derived from embryonic stem cells, Biochem. Biophys. Res. Commun. 461 (2015) 281-286, http://dx.doi.org/10.1016/j.bbrc.2015.04.018.

[43] K.A. Nave, G. Lemke, Induction of the myelin proteolipid protein (PLP) gene in C6 glioblastoma cells: functional analysis of the PLP promoter, J. Neurosci. 11 (1991) 3060-3069.

[44] V. Jaquet, G. Pfend, M. Tosic, J.M. Matthieu, Analysis of cis-acting sequences from the myelin oligodendrocyte glycoprotein promoter, J. Neurochem. 73 (1999) 120-128, http://dx.doi.org/10.1046/j.1471-4159.1999.0730120.x.

[45] F.S. Afshari, A.K. Chu, C. Sato-Bigbee, Effect of cyclic AMP on the expression of myelin basic protein species and myelin proteolipid protein in committed oligodendrocytes: differential involvement of the transcription factor CREB, J. Neurosci. Res. 66 (2001) 37-45, http://dx.doi.org/10.1002/jnr.1195.

[46] A. Franko, S. Mayer, G. Thiel, L. Mercy L, T. Arnould, H.T. Hornig-Do, R.J. Wiesner, S. Goffart, CREB-1 alpha is recruited to and mediates upregulation of the cytochrome c promoter during enhanced mitochondrial biogenesis accompanying skeletal muscle differentiation, Mol. Cell. Biol. 28 (2008) 2446-2459, http://dx.doi.org/10.1128/MCB.00980-07.

[47] N. Ishiguro, G.D. Brown, A. Ishizu, D. Meruelo, The regulation of murine H2Dd expression by activation transcription factor 1 and cAMP response element binding protein, J. Immunol. 160 (1998) 5907-5914.

[48] G. Lupo, M. Bertacchi, N. Carucci, G. Augusti-Tocco, S. Biagioni, F. Cremisi, From pluripotency to forebrain patterning: an in vitro journey astride embryonic stem 
cells, Cell. Mol. Life Sci. 71 (2014) 2917-2930, http://dx.doi.org/10.1007/s00018014-1596-1.

[49] K. Guan, H. Chang, A. Rolletschek, A.M. Wobus, Embryonic stem cell-derived neurogenesis. Retinoic acid induction and lineage selection of neuronal cells, Cell Tissue Res. 305 (2001) 171-176, http://dx.doi.org/10.1007/s004410100416.

[50] T. Glaser, O. Brüstle, Retinoic acid induction of ES-cell-derived neurons: the radial glia connection. Trends Neurosci. 28 (2005) 397-400, http://dx.doi.org/10.1016/j.tins.2005.05.008.

[51] Q.L. Ying, J. Nichols, I. Chambers, A. Smith, BMP induction of Id proteins suppresses differentiation and sustains embryonic stem cell self-renewal in collaboration with STAT3, Cell 115 (2003) 281-292, http://dx.doi.org/10.1016/S0092-8674(03)00847-X.

[52] H.J. Park, M. Hong, R.T. Bronson, M.A. Israel, W. N. Frankel, and K. Yun, Elevated Id 2 expression results in precocious neural stem cell depletion and abnormal brain development, Stem Cells 31 (2013) 1010-1021,

http://dx.doi.org/10.1002/stem.1351.

[53] B. Fischer, K. Azim, A. Hurtado-Chong, S. Ramelli, M. Fernández, O. Raineteau, E-proteins orchestrate the progression of neural stem cell differentiation in the postnatal forebrain, Neural Dev. 9 (2014) 23, http://dx.doi.org/10.1186/17498104-9-23.

[54] A. Longo, G.P. Guanga, R.B. Rose, Crystal structure of E47-NeuroD1/beta2 bHLH domain-DNA complex: heterodimer selectivity and DNA recognition, Biochemistry 47 (2008) 218-229, http://dx.doi.org/10.1021/bi701527r.

[55] M.A. Schumacher, R.H. Goodman, R.G. Brennan, The structure of a CREB bZIP somatostatin CRE complex reveals the basis for selective dimerization and divalent cation-enhanced DNA binding, J. Biol. Chem. 275 (2000) 35242-35247, http://dx.doi.org/10.1074/jbc.M007293200.

[56] K. Langlands, X. Yin, G. Anand, E.V. Prochownik, Differential interactions of Id proteins with basic-helix-loop-helix transcription factors, J. Biol. Chem. 272 (1997) 19785-19793, http://dx.doi.org/10.1074/jbc.272.32.19785.

[57] T. Muir, J. Wilson-Rawls, J.D. Stevens, A. Rawls, R. Schweitzer, C. Kang, M.K. Skinner, Integration of CREB and bHLH transcriptional signaling pathways through 
direct heterodimerization of the proteins: role in muscle and testis development, Mol. Reprod. Dev. 75 (2008) 1637-1652, http://dx.doi.org/10.1002/mrd.20902.

[58] J. Samanta, J.A. Kessler, Interactions between ID and OLIG proteins mediate the inhibitory effects of BMP4 on oligodendroglial differentiation, Development, 131 (2004) 4131-4142, http://dx.doi.org/10.1242/dev.01273.

[59] X.S. Chen, Y.H. Zhang, Q.Y. Cai, Z.X. Yao, ID2: A negative transcription factor regulating oligodendroglia differentiation, J. Neurosci. Res. 90 (2012) 925932, http://dx.doi.org/10.1002/jnr.22826.

[60] S.P. Fancy, M.R. Kotter, E.P. Harrington, J.K. Huang, C. Zhao, D.H. Rowitch, R.J. Franklin, Overcoming remyelination failure in multiple sclerosis and other myelin disorders, Exp. Neurol. 225 (2010) 18-23, http://dx.doi.org/10.1016/j.expneurol.2009.12.020.

[61] K. Kitajka, A.J. Sinclair, R.S. Weisinger, H.S. Weisinger, M. Mathai, A.P. Jayasooriya, J.E. Halver, L.G. Puskás, Effects of dietary omega-3 polyunsaturated fatty acids on brain gene expression, Proc. Natl. Acad. Sci. U S A 101 (2004) 1093110936, http://dx.doi.org/10.1073/pnas.0402342101.

[62] D. Ma, M. Zhang, C.P. Larsen, F. Xu, W. Hua, T. Yamashima, Y. Mao, L. Zhou, DHA promotes the neuronal differentiation of rat neural stem cells transfected with GPR40 gene, Brain Res. 1330 (2010) 1-8, http://dx.doi.org/10.1016/j.brainres.2010.03.002.

[63] T. Yamashima, Dual effects of the non-esterified fatty acid receptor 'GPR40' for human health, Prog. Lipid Res. 58 (2015) 40-50, http://dx.doi.org/10.1016/j.plipres.2015.01.002.

[64] N.B. Boneva, T. Yamashima, New insights into "GPR40-CREB interaction in adult neurogenesis" specific for primates, Hippocampus 22 (2012) 896-905, http://dx.doi.org/10.1002/hipo.20951.

[65] M. Zamarbide, I. Etayo-Labiano, A. Ricobaraza, E. Martínez-Pinilla, M.S. Aymerich , J. Luis Lanciego, A. Pérez-Mediavilla, R. Franco, GPR40 activation leads to CREB and ERK phosphorylation in primary cultures of neurons from the mouse CNS and in human neuroblastoma cells, Hippocampus 24 (2014) 733-739, http://dx.doi.org/10.1002/hipo.22263. 
[66] K. Nakamoto, T. Nishinaka, K. Matsumoto, F. Kasuya, M. Mankura, Y.

Koyama, S. Tokuyama, Involvement of the long-chain fatty acid receptor GPR40 as a novel pain regulatory system, Brain Res. 1432 (2012) 74-83, http://dx.doi.org/10.1016/j.brainres.2011.11.012.

[67] J.J. Kohler, S.J. Metallo, T.L. Schneider, A. Schepartz, DNA specificity enhanced by sequential binding of protein monomers, Proc. Natl. Acad. Sci. U S A 96 (1999) 11735-11739, http://dx.doi.org/10.1073/pnas.96.21.11735.

[68] I.A. Qureshi, S. Gokhan, M.F. Mehler, REST and CoREST are transcriptional and epigenetic regulators of seminal neural fate decisions, Cell Cycle 9 (2010) 44774486, http://dx.doi.org/10.4161/cc.9.22.13973.

[69] R. Teachenor, K. Beck, L.Y.Wright, Z. Shen, S.P. Briggs, C. Murre, Biochemical and phosphoproteomic analysis of the helix-loop-helix protein E47, Mol. Cell. Biol. 32 (2012) 1671-1682, http://dx.doi.org/10.1128/MCB.06452-11.

[70] S. San-Marina, Y. Han, F. Suarez Saiz, M.R. Trus, M.D. Minden, Lyl1 interacts with CREB1 and alters expression of CREB1 target genes, Biochim. Biophys. Acta 1783 (2008) 503-517, https://dx.doi.org/10.1016/j.bbamcr.2007.11.015.

[71] S. San-Marina, Y. Han, J. Liu, M.D. Minden, Suspected leukemia oncoproteins CREB1 and LYL1 regulate Op18/STMN1 expression, Biochim. Biophys. Acta 1819 (2012) 1164-1172, http://dx.doi.org/10.1016/j.bbagrm.2012.09.004.

[72] L.J. Everett, J. Le Lay, S. Lukovac, D. Bernstein, D.J. Steger, M.A. Lazar, K.H. Kaestner, Integrative genomic analysis of CREB defines a critical role for transcription factor networks in mediating the fed/fasted switch in liver, BMC Genomics 14 (2013) 337, http://dx.doi.org/10.1186/1471-2164-14-337.

[73] E. Benito, L.M. Valor, M. Jimenez-Minchan, W. Huber, A. Barco, cAMP response element-binding protein is a primary hub of activity-driven neuronal gene expression, J. Neurosci. 31 (2011) 18237-18250, http://dx.doi.org/10.1523/JNEUROSCI.4554-11.2011.

[74] S. Wang, A. Sdrulla, J.E. Johnson, Y. Yokota, B.A. Barres, A role for the helixloop-helix protein Id 2 in the control of oligodendrocyte development, Neuron 29 (2001) 603-614, http://dx.doi.org/10.1016/S0896-6273(01)00237-9.

[75] A. Lasorella, R. Benezra, A. Iavarone, The ID proteins: master regulators of cancer stem cells and tumour aggressiveness, Nat. Rev. Cancer 14 (2014) 77-91, http://dx.doi.org/10.1038/nrc3638. 
197 [76] C. Roschger, C. Cabrele, The Id-protein family in developmental and cancer198 associated pathways, Cell Commun. Signal, 15 (2017) 7, 199 http://dx.doi.org/10.1186/s12964-016-0161-y.

200 [77] M. Simons, K.A. Nave, Oligodendrocytes: Myelination and Axonal Support, 201 Cold Spring Harb. Perspect. Biol. Pii (2015) a020479, 202 http://dx.doi.org/10.1101/cshperspect.a020479. 
Supplemental Figure S1

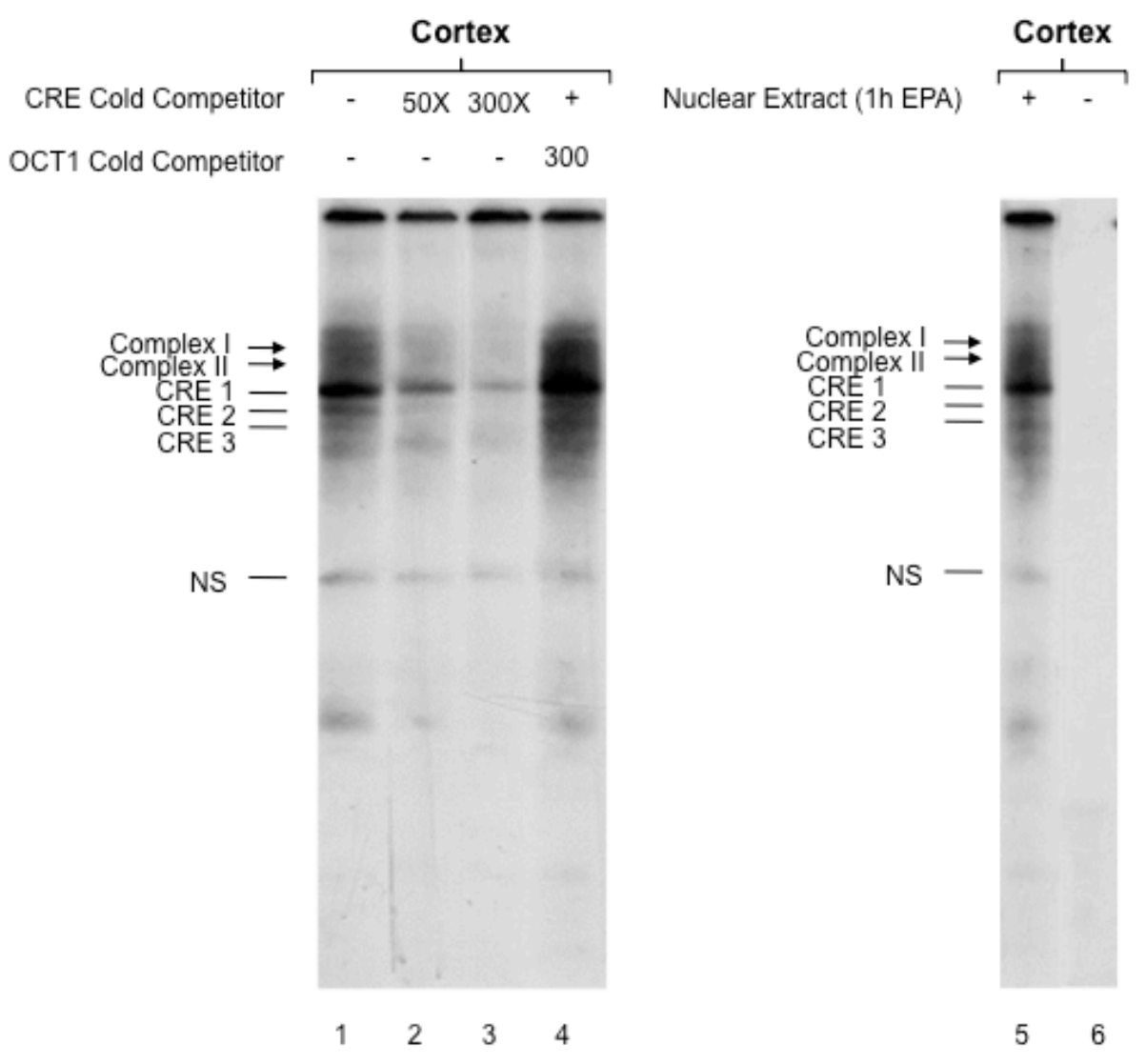

224 Supplementary Fig. S1. EMSA analysis of CRE DNA-binding activity of nuclear 225 extracts from cortex in rat pups treated with EPA for $1 \mathrm{~h}$. Sequence specificity of .226 binding was verified by the addition of 50 -fold and 300 -fold molar excess of CRE 227 cold competitor oligonucleotides (lanes 2 and 3) or in the presence of 300 -fold molar .228 excess of the non-CRE containing OCT-1 oligonucleotides, as negative control (lane .229 4). Lanes 5 and 6 , positive and negative controls with or without nuclear extracts. NS, 230 are nonspecific bands. 
A

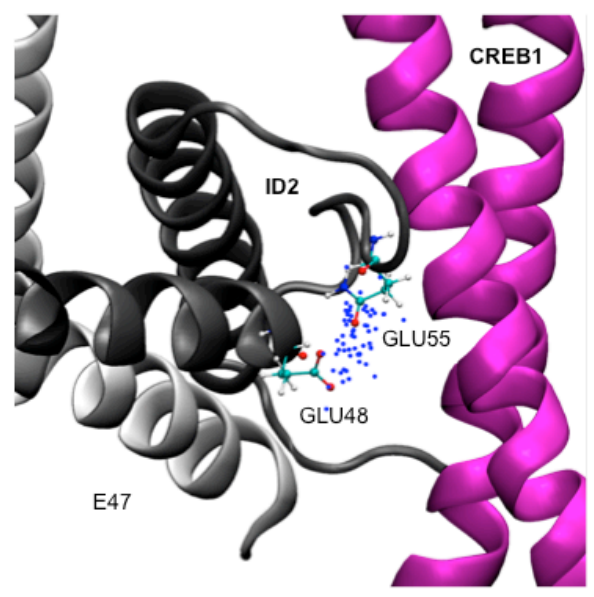

B 
Supplemental Table 1: Optimization of the interface area between the ID2:E47 unit and the CREB1 homodimer for rotations around the axes of Fig. 6A.

\begin{tabular}{|c|c|c|c|c|c|c|c|}
\hline $\begin{array}{l}\text { Rotation angle } \\
\text { for left axis } \\
\text { (deg) }\end{array}$ & $\begin{array}{l}\text { Rotation angle } \\
\text { for right axis } \\
\quad(\mathrm{deg})\end{array}$ & $\begin{array}{c}\text { Size of } \\
\text { interface }\left(\AA^{2}\right)\end{array}$ & $\begin{array}{c}\text { Minimal C } \alpha- \\
\text { C } \alpha \text { distance } \\
(\AA)\end{array}$ & $\begin{array}{l}\text { Rotation angle } \\
\text { for left axis } \\
\text { (deg) }\end{array}$ & $\begin{array}{l}\text { Rotation angle } \\
\text { for right axis } \\
\text { (deg) }\end{array}$ & $\begin{array}{c}\text { Size of } \\
\text { interface }\left(\AA^{2}\right)\end{array}$ & $\begin{array}{c}\text { Minimal C } \alpha \text { - } \\
\text { C } \alpha \text { distance } \\
(\AA)\end{array}$ \\
\hline-60 & -60 & 820 & 1,3 & 0 & 0 & 922 & 4,2 \\
\hline-60 & -45 & 807 & 2,7 & 0 & 15 & 890 & 3,7 \\
\hline-60 & -30 & 871 & 4,3 & 0 & 30 & 803 & 0,7 \\
\hline-60 & -15 & 945 & 4,0 & 0 & 45 & 777 & 1,6 \\
\hline-60 & 0 & 964 & 3,1 & 0 & 60 & 765 & 0,8 \\
\hline-60 & 15 & 940 & 2,2 & 15 & -60 & 785 & 2,2 \\
\hline-60 & 30 & 807 & 1,7 & 15 & -45 & 769 & 2,8 \\
\hline-60 & 45 & 751 & 1,6 & 15 & -30 & 820 & 2,9 \\
\hline-60 & 60 & 759 & 1,7 & 15 & -15 & 855 & 4,1 \\
\hline-45 & -60 & 811 & 2,2 & 15 & 0 & 885 & 3,7 \\
\hline-45 & -45 & 789 & 4,0 & 15 & 15 & 848 & 4,2 \\
\hline-45 & -30 & 876 & 5,3 & 15 & 30 & 833 & 3,0 \\
\hline-45 & -15 & 1000 & 4,2 & 15 & 45 & 740 & 0,5 \\
\hline-45 & 0 & 998 & 3,5 & 15 & 60 & 697 & 2,2 \\
\hline-45 & 15 & 941 & 2,8 & 30 & -60 & 796 & 1,1 \\
\hline-45 & 30 & 819 & 1,7 & 30 & -45 & 749 & 2,8 \\
\hline-45 & 45 & 740 & 1,9 & 30 & -30 & 790 & 3,0 \\
\hline-45 & 60 & 778 & 1,3 & 30 & -15 & 796 & 4,0 \\
\hline-30 & -60 & 839 & 3,3 & 30 & 0 & 806 & 2,5 \\
\hline-30 & -45 & 842 & 4,2 & 30 & 15 & 759 & 4,5 \\
\hline-30 & -30 & 927 & 5,8 & 30 & 30 & 834 & 3,8 \\
\hline-30 & -15 & 1012 & 4,9 & 30 & 45 & 682 & 1,7 \\
\hline-30 & 0 & 965 & 4,5 & 30 & 60 & 596 & 1,3 \\
\hline-30 & 15 & 896 & 3,4 & 45 & -60 & 804 & 1,2 \\
\hline-30 & 30 & 796 & 2,5 & 45 & -45 & 747 & 2,6 \\
\hline-30 & 45 & 734 & 0,5 & 45 & -30 & 761 & 2,1 \\
\hline-30 & 60 & 797 & 1,8 & 45 & -15 & 756 & 4,2 \\
\hline-15 & -60 & 841 & 3,0 & 45 & 0 & 734 & 2,7 \\
\hline-15 & -45 & 863 & 3,4 & 45 & 15 & 678 & 4,0 \\
\hline-15 & -30 & 928 & 4,9 & 45 & 30 & 761 & 5,1 \\
\hline-15 & -15 & 986 & 5,3 & 45 & 45 & 644 & 3,1 \\
\hline-15 & 0 & 935 & 4,8 & 45 & 60 & 538 & 3,2 \\
\hline-15 & 15 & 870 & 3,5 & 60 & -60 & 817 & 2,4 \\
\hline-15 & 30 & 781 & 1,8 & 60 & -45 & 757 & 2,0 \\
\hline-15 & 45 & 770 & 1,1 & 60 & -30 & 766 & 2,2 \\
\hline-15 & 60 & 787 & 1,7 & 60 & -15 & 759 & 4,3 \\
\hline 0 & -60 & 809 & 2,2 & 60 & 0 & 716 & 3,9 \\
\hline 0 & -45 & 824 & 3,5 & 60 & 15 & 666 & 3,0 \\
\hline 0 & -30 & 852 & 3,7 & 60 & 30 & 634 & 4,9 \\
\hline 0 & -15 & 943 & 4,4 & 60 & 45 & 606 & 4,5 \\
\hline 0 & 0 & 922 & 4,2 & 60 & 60 & 583 & 4,2 \\
\hline
\end{tabular}

254 Legend of Supplementary Table 1: The size of the interface between the ID2:47

.255 unit and the CREB1 homodimer is listed as function of the angles of rotation. A

256 negative sign indicates left-handed rotation. The starting point of the optimization

$257\left(0^{\circ}, 0^{\circ}\right)$ and the entry with maximal interface size $\left(-30^{\circ},-15^{\circ}\right)$ are marked by frames.

258 Furthermore, the minimal distance between the backbone $\mathrm{C} \alpha$ atoms of the CREB1

259 dimer and the ID2:E47 complex is given. Combinations of rotation angles that do not

260 lead to a steric clash are highlighted in grey. 
261 Legend of Supplementary Video 1. A movie showing distinct $\mathrm{Ca}^{2+}$ binding sites 262 identified in the $[\mathrm{CREB} 1]_{2}:$ ID2: E47 tetramer. An overlay of all calcium positions 263 sampled during a $500 \mathrm{~ns}$ molecular dynamics simulation reveals the most likely .264 calcium binding sites (blue cloud). The color code is as follows: CREB1: magenta; 265 ID2: gray; E47: white; DNA: black; calcium ions: blue dots.

266 